\title{
Robust Neighbor Discovery in Multi-Hop Multi-Channel Heterogeneous Wireless Networks*
}

\author{
Yanyan Zeng` K. Alex Mills, Shreyas Gokhale, Neeraj Mittal; S. Venkatesan, and \\ R. Chandrasekaran \\ Department of Computer Science \\ The University of Texas at Dallas \\ Richardson, TX 75080, USA \\ E-mails: yanyan.bansal@gmail.com k.alex.mills@utdallas.edu \\ shreyas.gokhale@utdallas.edu neerajm@utdallas.edunvenky@utdallas.edu \\ chandra@utdallas.edu
}

\begin{abstract}
An important first step when deploying a wireless ad hoc network is neighbor discovery in which every node attempts to determine the set of nodes it can communicate within one wireless hop. In the recent years, cognitive radio $(C R)$ technology has gained attention as an attractive approach to alleviate spectrum congestion. A cognitive radio transceiver can operate over a wide range of frequencies possibly spanning multiple frequency bands. A cognitive radio node can opportunistically utilize unused wireless spectrum without interference from other wireless devices in its vicinity. Due to spatial variations in frequency usage and hardware variations in radio transceivers, different nodes in the network may perceive different subsets of frequencies available to them for communication. This heterogeneity in the available channel sets across the network increases the complexity of solving the neighbor discovery problem in a cognitive radio network. In this work, we design and analyze several randomized algorithms for neighbor discovery in such a (heterogeneous) network under a variety of assumptions (e.g., maximum node

${ }^{*}$ A preliminary version of the work has appeared earlier in the Proceedings of the 31st International Conference on Distributed Computing Systems (ICDCS), 2011 [45].

${ }^{\dagger}$ Yanyan Zeng is currently working at JPMorgan Chase.

${ }^{\ddagger}$ This work was supported, in part, by the National Science Foundation (NSF) under grant number CNS-1115733.
\end{abstract}


degree known or unknown) for both synchronous and asynchronous systems under minimal knowledge. We also show that our randomized algorithms are naturally suited to tolerate unreliable channels and adversarial attacks.

Key words: multi-hop multi-channel wireless network, cognitive radio technology, heterogeneous channel availability, neighbor discovery, randomized algorithm, asynchronous system, clock drift, lossy channel, jamming attack 


\section{Introduction}

Neighbor discovery is an important step in forming a self-organizing wireless ad hoc network without any support from an existing communication infrastructure $[8,9,19,20,29,42,51,54,57,58,63]$. When deployed, nodes initially have no prior knowledge of other nodes that they can communicate with directly.

The results of neighbor discovery can be used to solve other important communication problems such as medium access control [7,22], routing [15], broadcasting [50,54], clustering [16, 32, 40], collision-free scheduling [26, 27], spanning tree construction [21], and topology control [37,62]. Many algorithms for solving these problems implicitly assume that all nodes know their one-hop and sometimes even two-hop neighbors. Many location-based routing protocols (e.g. localized routing in Vehicular Ad hoc Networks (VANETs)) use the position of neighboring nodes to make routing/forwarding decisions. The neighborhood information is also used to update the reachability status of nodes. A better neighbor discovery algorithm, which uses fewer messages and has higher accuracy, can be used to improve the performance of locationbased routing algorithms [10-12]. Neighborhood information helps reduce the cost of flooding in multicast tree construction using flooding algorithms [39]. Mobile sensing applications ranging from mobile social networking to proximity-based gaming involves collection and sharing of data among nearby users. The success of these applications depends on neighborhood information [20]. Neighbor discovery is extremely important in Underwater Acoustic (UWA) Networks and needs to be done frequently because nodes may move proactively due to the unpredictable underwater currents [68]. More details of how the results of neighbor discovery can be used to solve other communication problems can be found in $[10,11]$.

Cognitive radio (CR) technology has emerged as a promising approach for improving spectrum utilization efficiency and meeting the increased demand for wireless communications [13]. A CR node can scan a part of the wireless spectrum, and identify unused or underutilized channels in the spectrum $[1,65]$. CR nodes in a network can then use these channels opportunistically for communication among themselves even if the channels belong to licensed users. The licensed users are referred to as the primary users, and CR nodes are referred to as the secondary users. (Of course, when a primary user arrives and starts using its channel, the secondary users have to vacate the channel.) Due to spatial variations in frequency usage, hardware variations in radio transceivers and uneven propagation of wireless signals, different nodes in the network may perceive different subsets of frequencies available to them for communication. This gives rise to a multi-hop, multi-channel, heterogeneous wireless network, abbreviated as $M^{2} H e W n e t w o r k$. The focus 
of this work is on solving the neighbor discovery problem in an $\mathrm{M}^{2} \mathrm{HeW}$ network.

A large number of neighbor discovery algorithms have been proposed in the literature. Most of the algorithms suffer from one or more of the following limitations: (i) all nodes are assumed to be synchronized (synchronous system), (ii) the entire network is assumed to operate on a single channel (single-channel network), (iii) all nodes are assumed to be able to communicate with each other (single-hop network), (iv) all channels are assumed to be available to all nodes (homogeneous network), or (v) the algorithm is only evaluated experimentally (no theoretical guarantees provided). A more detailed discussion of the related work can be found in Section 7.

Our Contributions: Our main contribution in this work is two randomized neighbor discovery algorithms for an $M^{2} \mathrm{HeW}$ network when the system is asynchronous that guarantee success with arbitrarily high probability. The first algorithm assumes that nodes know a good upper bound on the maximum degree of any node in the network. The second algorithm does not make any such assumption. Both algorithms only assume that the maximum drift rate of the clock of any node is bounded, with the second algorithm assuming a tighter but unknown bound whose value depends on various system parameters. None of the algorithms require clocks of different nodes to be synchronized. In fact, clocks of any two nodes may have arbitrary skew with respect to each other. Other advantages of our algorithms are as follows: (i) nodes do not need to agree on a universal channel set, and (ii) the running time of an algorithm depends on the "degree of heterogeneity" in the network; the running time decreases as the available channel sets become more homogeneous.

Our algorithms for an asynchronous system are based on those for a synchronous system. Therefore, as additional contributions, we also present a suite of randomized neighbor discovery algorithms for an $M^{2} \mathrm{HeW}$ network when the system is synchronous under a variety of assumptions such as: (i) whether all the nodes start executing the neighbor discovery algorithm at the same time or not, and (ii) whether nodes are aware of an estimate on the upper bound on the maximum degree of any node in the network or not.

We believe that our approach for transforming a state-less algorithm developed for a synchronous $\mathrm{M}^{2} \mathrm{HeW}$ network to work for an asynchronous $\mathrm{M}^{2} \mathrm{HeW}$ network can also be applied to other important communication problems in an $\mathrm{M}^{2} \mathrm{HeW}$ network with running time increasing by only a constant factor.

We show that our randomized algorithms can easily tolerate unreliable channels. We also prove that, our algorithms, with minor modification in the asynchronous case, are tolerant to jamming attacks by a reactive but "memory-less" jammer under certain assumption. The running time of our algorithms, when subject 
to a jamming attack, increases by at most a constant factor. In fact, for sufficiently large values of system parameters (namely, number of nodes and number of channels), the running time increases only by a factor of at most two in the worst-case.

Organization: The rest of the manuscript is organized as follows. We describe our system model for a multi-hop multi-channel heterogeneous wireless network in Section 2. For ease of exposition, we first present a suite of randomized neighbor discovery algorithms for a synchronous system under a variety of assumptions and analyze their time complexity in Section 3. We then present two randomized neighbor discovery algorithms for an asynchronous system, which are derived from their synchronous counterparts, and analyze their complexity in Section 4. We discuss several extensions to our algorithms to enhance their applicability and improve their robustness in Section 6. Finally, in Section 7, we give a comparison of our contributions with existing research and also examine other related work done on neighbor discovery.

\section{System Model}

We assume a multi-hop multi-channel heterogeneous wireless $\left(\mathrm{M}^{2} \mathrm{HeW}\right)$ network consisting of one or more radio nodes. Let $N$ denote the total number of radio nodes. Nodes do not know $N$. Each radio node is equipped with a transceiver (transmitter-receiver pair), which is capable of operating over multiple frequencies or channels. However, at any given time, a transceiver can operate (either transmit or receive) over a single channel only. Further, a transceiver cannot transmit and receive at the same time. Transceivers of different nodes need not be identical; the set of channels over which a transceiver can operate may be different for different transceivers.

Different nodes in a network may have different sets of channels available for communication. For example, in a cognitive radio network (a type of $\mathrm{M}^{2} \mathrm{HeW}$ network), each node can scan the frequency spectrum and identify the subset of unused or under-used portions of the spectrum even those that have been licensed to other users or organizations [13]. A node can potentially use such frequencies to communicate with its neighbors until they are reclaimed by their licensed (primary) users [13]. Due to spatial variations in frequency usage/interference and hardware variations in radio transceivers, different nodes in the network may perceive different subsets of frequencies available to them for communication. We refer to the subset of frequencies or channels that a node can use to communicate with its neighbors as the available channel 
set of the node. For a node $u$, we use $\mathcal{A}(u)$ to denote its available channel set. We use $S$ to denote the size of the largest available channel set, that is, $S=\max _{u}|\mathcal{A}(u)|$. Note that nodes do not know $S$.

We say that a node $v$ is a neighbor of node $u$ on a channel $c$ if $u$ can reliably receive any message transmitted by $v$ on $c$ provided no other node in the network is transmitting on $c$ at the same time, and vice versa. We assume that the communication graph is symmetric because unidirectional neighborhood relationships are expensive and impractical to use in wireless networks [47] (although our algorithms work for asymmetric communication graphs as well). For a node $u$ and a channel $c \in \mathcal{A}(u)$, we use $\Delta(u, c)$ to denote the number of neighbors, also known as degree, of $u$ on $c$. We use $\Delta$ denote the maximum degree of any node on any channel, that is, $\Delta=\max _{u} \max _{c \in \mathcal{A}(u)} \Delta(u, c)$.

Note that, if nodes $u$ and $v$ are neighbors of each other on some channel, then $u$ has to discover $v$ and $v$ has to discover $u$ separately. It is convenient to assume two separate links—one from $u$ to $v$ and another from $v$ to $u$. We use $(u, v)$ to denote the link from $u$ to $v$. We refer to the set of channels on which the link $(u, v)$ can operate as the span of $(u, v)$ and denote it by $\operatorname{span}(u, v)$. Note that $\operatorname{span}(u, v) \subseteq \mathcal{A}(u) \cap \mathcal{A}(v)$. We refer to the ratio of $|\operatorname{span}(u, v)|$ to $|\mathcal{A}(v)|$ as the span-ratio of the link $(u, v)$. Note that the span-ratio of any link lies between $\frac{1}{S}$ and 1 . Further, the span-ratio of $(u, v)$ may be different from the span-ratio of $(v, u)$ (because $|\mathcal{A}(u)|$ may be different from $|\mathcal{A}(v)|$ ). We use $\rho$ to denote the minimum span-ratio among all links. Note that nodes do not know $\rho$. Intuitively, $\rho$ can be viewed as a measure of the degree of homogeneity in the network - the larger the value, the more homogeneous the network is in terms of channel availability for nodes and links. The running time of our algorithms is inversely proportional to $\rho$. When all links can operate on all available channels (an assumption made frequently in the literature), $\rho=1$, which minimizes the running time of our algorithms. In general, the more heterogeneous the network is, the larger is the running time of our algorithms.

If nodes $v$ and $w$ are neighbors of node $u$ on channel $c$ and both $v$ and $w$ transmit on $c$ at the same time, then their transmissions collide at $u$. If $u$ is listening on $c$ when $v$ and $w$ transmitting on $c$ at the same time, then $u$ hears only noise. We do not assume that nodes can detect collisions, that is, distinguish between background noise and collision noise.

The set of channels over which an $\mathrm{M}^{2} \mathrm{HeW}$ network can operate may have very different propagation characteristics. For example, a cognitive radio transceiver may be capable of operating over a wide range of frequencies (e.g., as low as $100 \mathrm{MHz}$ to as high as $2.5 \mathrm{GHz}$ [14]). Radio waves at different frequencies undergo different angles of refraction and therefore propagate in different ways. As a result, two radio nodes 
$u$ and $v$ that are neighbors on some channel $c$ may not be neighbors on another channel $d$ even if both $u$ and $v$ have $d$ in their available channel sets. This may happen, for example, if $c$ is a channel in the 900 $\mathrm{MHz}$ band and $d$ is a channel in the $2.4 \mathrm{GHz}$ band; a lower frequency has stronger capability to penetrate through different materials and therefore has a longer transmission range than a higher frequency. For ease of exposition, we first describe our algorithms assuming that all frequencies or channels have identical propagation characteristics. This implies that if a communication link from node $u$ to node $v$ can operate over some channel $c \in \mathcal{A}(u) \cap \mathcal{A}(v)$ then it can also operate over all channels in $\mathcal{A}(u) \cap \mathcal{A}(v)$. We later discuss how to extend our algorithms to handle diverse propagation characteristics of different frequencies.

We use "log" to refer to the logarithm to the base 2 and "ln" to refer to the natural logarithm. In this paper, we investigate the neighbor discovery problem both when the system is synchronous and when the system is asynchronous. We now describe how we model each type of system.

Synchronous System: We assume that the execution of the system is divided into synchronized time-slots. In each time slot, each node can be in one of the following three modes: (i) transmit mode on some channel in its available channel set, (ii) receive mode on some channel in its available channel set, or (iii) quiet mode when the transceiver is shut-off. Nodes may or may not start at the same time.

Asynchronous System: We assume that every node is equipped with a clock. Clocks of different nodes are not required to be synchronized. For a clock $C$ and time $t$, we use $C(t)$ to denote the value of $C$ at $t$. For a node $u$, we use $C_{u}$ to denote the clock of $u$. The clock of a node may have non-zero drift and the drift rate may change over time. The drift rate of a clock $C$ at time $t$ is given by $\frac{d C}{d t}-1$. If $C$ is an ideal clock, then, $\forall t, \Delta t \geq 0, C(t+\Delta t)-C(t)=\Delta t$; thus $\frac{d C}{d t}=1$. At any given time, different clocks may have different drift rates. Further, the drift rate of the same clock may change over time both in magnitude and sign. We do, however, assume that the magnitude of the maximum drift rate is bounded and let the bound be denoted by $\delta$. This implies that $\forall t, \Delta t \geq 0$,

$$
(1-\delta) \Delta t \leq C(t+\Delta t)-C(t) \leq(1+\delta) \Delta t
$$

For an ideal clock, $\delta=0$. In practice, $\delta$ is quite small (e.g., $10^{-6}$ seconds/second for an quartz clock). 


\section{Neighbor Discovery in Synchronous Systems}

We first describe our algorithms for neighbor discovery assuming that all nodes start executing the algorithm at the same time. We then relax this assumption and describe our algorithms for neighbor discovery when different nodes may start executing the algorithm at different times. Later, in Section 4, we use the algorithms for variable start times to derive randomized neighbor discovery algorithms for an asynchronous system.

\subsection{Identical Start Times}

We first assume that the nodes know some upper bound on maximum node degree and present a neighbor discovery algorithm. The bound need not be tight and, in fact, may be quite loose (since the dependence is logarithmic on the value of the upper bound). But all nodes should agree on a common upper bound. We then relax this restriction and describe an algorithm for the case when such knowledge is not available.

\subsubsection{Knowledge of Loose Upper Bound on Maximum Node Degree}

Let $\Delta_{\text {est }}$ denote an upper bound on the maximum node degree as known to all nodes. The execution of the algorithm is divided into stages. Each stage consists of $\left\lceil\log \left(\Delta_{\text {est }}\right)\right\rceil$ time-slots. In each time-slot of a stage, a node randomly chooses a channel from its available channel set, estimates its own degree (which may be quite different from the maximum degree) using doubling technique, and transmits on the chosen channel with a certain probability (and listens on that channel with the remaining probability). Specifically, in time-slot $i$ of a stage, where $1 \leq i \leq\left\lceil\log \left(\Delta_{e s t}\right)\right\rceil$, a node $u$ transmits on the selected channel, say $c$, with probability $\min \left(\frac{1}{2}, \frac{|\mathcal{A}(u)|}{2^{i}}\right)$ and listens on $c$ with the remaining probability.

A formal description of the algorithm is shown in Algorithm 1. It can be shown that, once a node's estimate of its own degree becomes close to its actual degree, the probability that it discovers one of its neighbors becomes significant. Consider a node $u$ and let node $v$ be its neighbor on a channel $c$. Observe that $1 \leq \Delta(u, c) \leq \Delta \leq \Delta_{\text {est }}$. Let $k=\max (1,\lceil\log \Delta(u, c)\rceil)$. Clearly, we have:

$$
2^{k-1} \leq \Delta(u, c) \leq 2^{k}
$$

We say that a time-slot $t$ covers the link $(v, u)$ on channel $c$ if during $t$ : (i) $v$ transmits on $c$, (ii) $u$ listens 


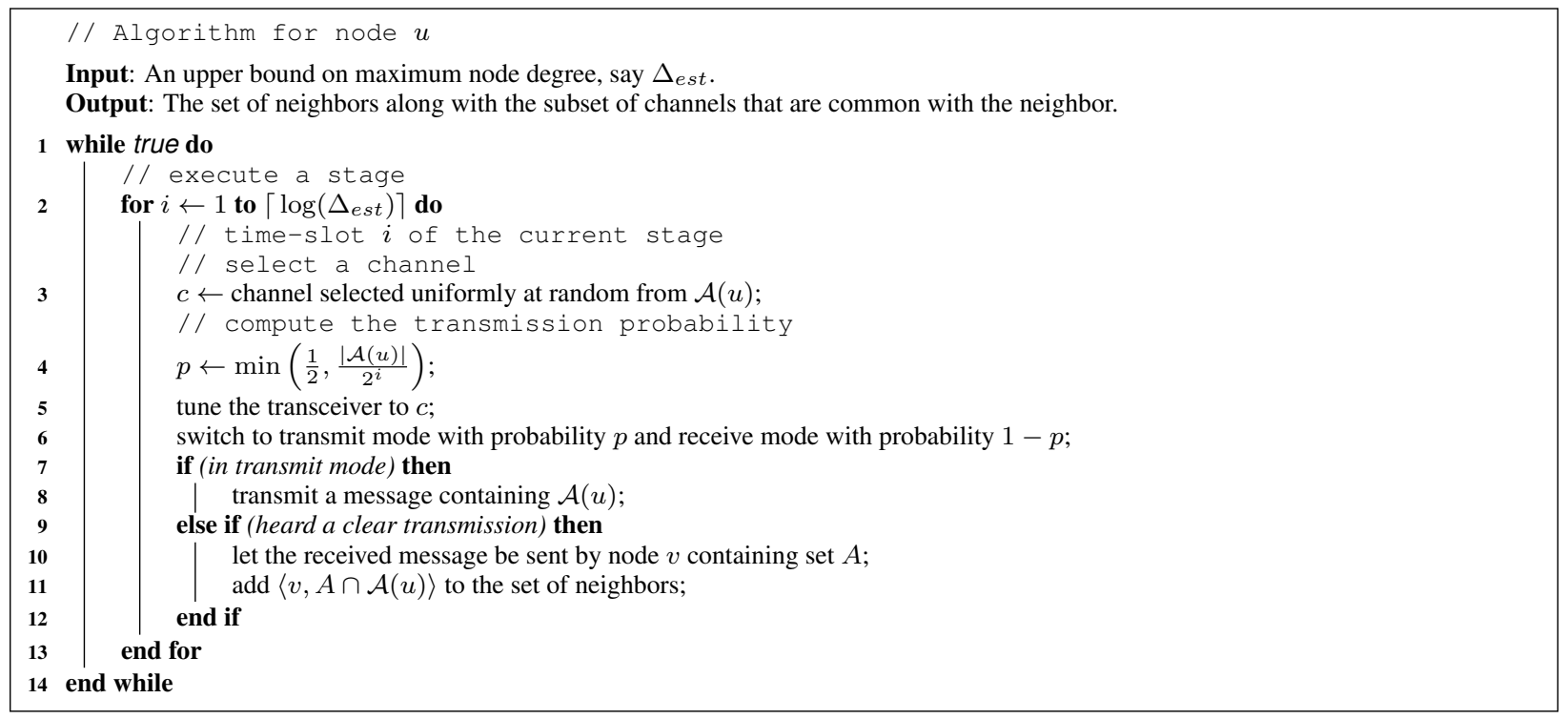

Algorithm 1: Neighbor discovery algorithm for a synchronous system with identical start times and knowledge of upper bound on maximum node degree.

on $c$, and (iii) no other neighbor of $u$ transmits on $c$. The three conditions collectively ensure that $u$ receives a clear message from $v$ (on $c$ during $t$ ). We say that a stage $s$ covers the link $(v, u)$ on channel $c$ if some time-slot of $s$ covers $(v, u)$ on $c$; also, $s$ covers $(v, u)$ if $s$ covers $(v, u)$ on some $c \in \operatorname{span}(v, u)$. Finally, we say that a sequence of stages covers the link $(v, u)$ if some stage of the sequence covers $(v, u)$.

Consider a stage $s$ and let $\tau$ denote the time-slot of $s$ that satisfies (2). We first compute the probability that $(v, u)$ is covered on $c$ during $\tau$. Let $A(\tau, c), B(\tau, c), C(\tau, c)$ denote the three events corresponding to the three conditions (i), (ii) and (iii), respectively, required for coverage. Note that the three events are mutually independent. We first compute the probability of occurrence of events $A(\tau, c), B(\tau, c)$ and $C(\tau, c)$ separately. We then compute the probability that $s$ covers $(v, u)$. The steps involved in computing the probabilities are standard and have been moved to the appendix (see Section A). We only state the results here. We have:

$$
\begin{aligned}
\operatorname{Pr}\{A(\tau, c)\} & \geq \frac{1}{2 \max (S, \Delta)} \\
\operatorname{Pr}\{B(\tau, c)\} & \geq \frac{1}{2|\mathcal{A}(u)|} \\
\operatorname{Pr}\{C(\tau, c)\} & \geq \frac{1}{4} \\
\operatorname{Pr}(s \text { covers }(v, u)) & \geq \frac{\rho}{16 \max (S, \Delta)}
\end{aligned}
$$




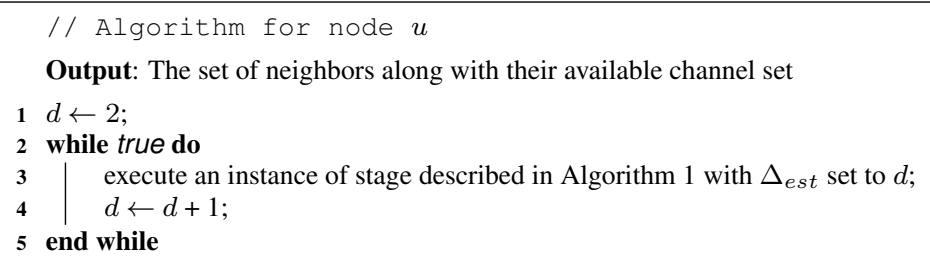

Algorithm 2: Neighbor discovery algorithm for a synchronous system with identical start times and no knowledge of maximum node degree.

Now, consider a sequence of $M=\frac{16 \max (S, \Delta)}{\rho} \ln \left(\frac{N^{2}}{\epsilon}\right)$ stages, where $\epsilon$ is the probability that neighbor discovery does not complete successfully. We show that the probability that the link $(v, u)$ is not covered within $M$ stages is at most $\frac{\epsilon}{N^{2}}$. The steps for computing the probability are fairly standard and have been moved to the appendix (see Section A). Formally,

$$
\operatorname{Pr}((v, u) \text { is not covered within } M \text { stages }) \leq \frac{\epsilon}{N^{2}}
$$

Finally, we have:

$$
\begin{aligned}
\operatorname{Pr}\left(\begin{array}{c}
\text { neighbor discovery does not finish } \\
\text { within } M \text { stages }
\end{array}\right) & =\operatorname{Pr}(\text { some link is not covered within } M \text { stages }) \\
& \leq \text { (number of links in the network) } \times \frac{\epsilon}{N^{2}} \leq \epsilon
\end{aligned}
$$

Therefore, we have the following theorem:

Theorem 1. Algorithm 1 guarantees that each node discovers all its neighbors on all channels within $O\left(\frac{\max (S, \Delta)}{\rho} \log \left(\Delta_{\text {est }}\right) \log \left(\frac{N}{\epsilon}\right)\right)$ time-slots with probability at least $1-\epsilon$.

\subsubsection{No Knowledge of Maximum Node Degree}

When knowledge about maximum node degree is not available, we employ the approach used by Nakano and Olariu in [46]. Starting with an estimate of two, we repeatedly execute an instance of stage with sequentially increasing values for the estimate (2, 3, 4, 5 and so on).

A formal description of the algorithm is shown in Algorithm 2. It can be easily verified that, once $d$ becomes greater than or equal to $\Delta$, each stage thereafter is guaranteed to contain a time-slot that satisfies (2) for every node. Recall that such time-slots are desirable because the probability that a node discovers one 
of its neighbors during such a time-slot is significant. To reach the success probability of at least $1-\epsilon$, from the analysis of Algorithm 1, it suffices for an execution to contain $M=\frac{16 \max (S, \Delta)}{\rho} \ln \left(\frac{N^{2}}{\epsilon}\right)$ stages, each of which consists of a time-slot that satisfies (2). In other words, neighbor discovery completes successfully with probability at least $1-\epsilon$ within $\Delta+M$ stages. Note that $M=\Omega(\Delta)$. Therefore we have:

Theorem 2. Algorithm 2 guarantees that each node discovers all its neighbors on all channels within $O(M \log M)$ time-slots with probability at least $1-\epsilon$, where $M=\frac{16 \max (S, \Delta)}{\rho} \ln \left(\frac{N^{2}}{\epsilon}\right)$.

\subsection{Variable Start Times}

We first assume that the nodes know some upper bound on maximum node degree and present a neighbor discovery algorithm. We then relax this restriction and describe an algorithm for the case when such a knowledge is not available. Let $T_{s}$ denote the time by which all nodes have initiated neighbor discovery.

\subsubsection{Knowledge of Good Upper Bound on Maximum Node Degree}

We assume that nodes know a "good" upper bound on the maximum node degree. Although the algorithm works even if the upper bound is loose, the running time of the algorithm will increase since it depends linearly on the value of the upper bound.

The main idea behind our algorithm is to ensure that the transmission probability of a node is same for every time-slot (but may be different for different nodes due to varying channel availability sets). This allows us to establish a "good" lower bound on the probability that a given link is covered in a time-slot. Let $\Delta_{e s t}$ denote an upper bound on the maximum node degree as known to all nodes. In each time-slot, a node $u$ randomly selects a channel from its available channel set, say $c$. It then transmits on $c$ with probability

$\min \left(\frac{1}{2}, \frac{|\mathcal{A}(u)|}{\Delta_{\text {est }}}\right)$ and listens on $c$ with the remaining probability. A formal description of the algorithm is shown in Algorithm 3.

For the analysis of the running time, as in the case of Algorithm 1, we can compute the probability of occurrence of events $A(\tau, c), B(\tau, c)$ and $C(\tau, c)$. We have:

$$
\begin{aligned}
\operatorname{Pr}\{A(\tau, c)\} & =\frac{1}{|\mathcal{A}(v)|} \times \min \left(\frac{1}{2}, \frac{|\mathcal{A}(v)|}{\Delta_{\text {est }}}\right)=\frac{1}{\max \left\{2|\mathcal{A}(v)|, \Delta_{\text {est }}\right\}} \\
& \geq \frac{1}{\max \left(2 S, \Delta_{\text {est }}\right)}
\end{aligned}
$$




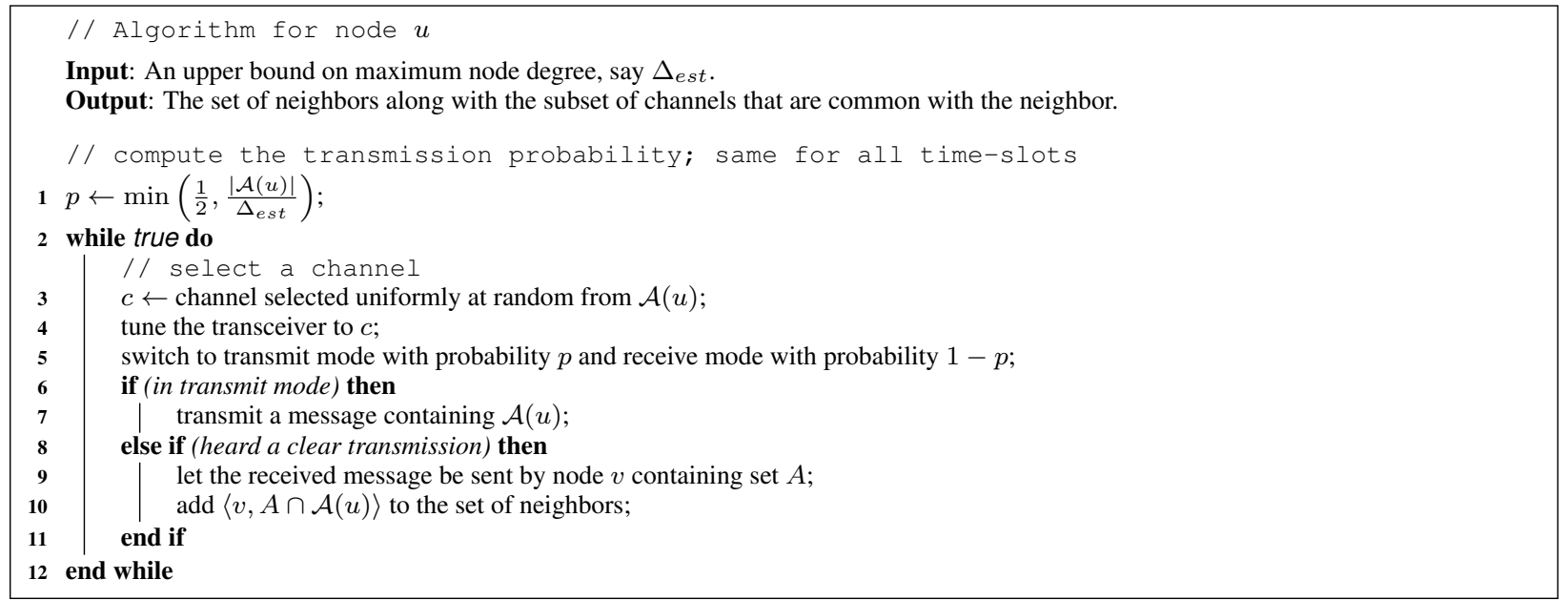

Algorithm 3: Neighbor discovery algorithm for a synchronous system with variable start times and knowledge of upper bound on maximum node degree.

It can be verified that the inequalities for $\operatorname{Pr}\{B(\tau, c)\}$ in (4) and $\operatorname{Pr}\{C(\tau, c)\}$ in (5) are still valid (although the proofs have to be slightly modified). Using an analysis similar to that for Algorithm 1, we can prove the following result:

Theorem 3. Algorithm 3 guarantees that each node discovers all its neighbors on all channels within $O\left(\frac{\max \left(2 S, \Delta_{\text {est }}\right)}{\rho} \log \left(\frac{N}{\epsilon}\right)\right)$ time-slots of $T_{s}$ with probability at least $1-\epsilon$.

Note that we no longer have a factor of $O\left(\log \left(\Delta_{\text {est }}\right)\right)$ in the time complexity because we do not have stages any more. However, we now require a tighter bound on the knowledge of maximum node degree.

\subsubsection{No Knowledge of Maximum Node Degree}

We refer to the difference in the start times of two nodes as slack, and use $\Theta$ to denote the maximum slack between any two nodes (expressed in terms of the number of time-slots). Also, note that no node knows $\Theta$. We present a neighbor discovery algorithm in which no node knows any of the other system parameters, namely, $S, \Delta, \rho, N$ and $\epsilon$. And, yet the algorithm completes neighbor discovery within a "short" period with high probability.

The main idea is to repeatedly execute instances of Algorithm 3 for different values of $\Delta_{\text {est }}$. The main challenge is to determine how long to run each instance of Algorithm 3 in order to achieve arbitrarily high success probability without knowing any of the system parameters. Let $T_{\text {est }}$ denote amount of time (in terms of number of slots) for which an instance of Algorithm 3 is run. We need to simultaneously estimate both 


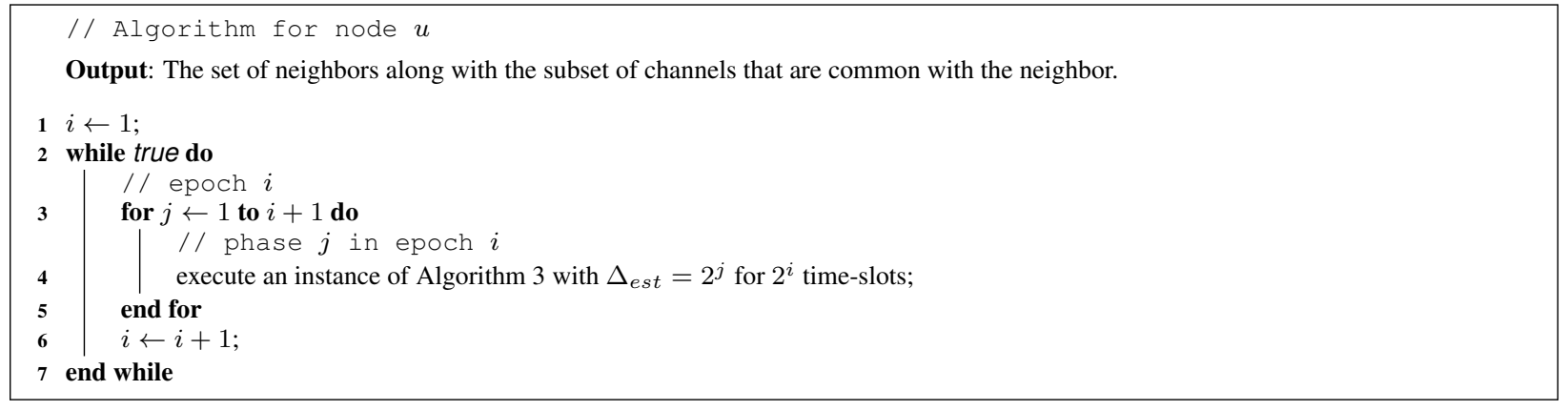

Algorithm 4: Neighbor discovery algorithm for a synchronous system with variable start times (no knowledge assumed).

$\Delta_{e s t}$ and $T_{e s t}$ so as to guarantee that neighbor discovery completes with arbitrarily high probability. To that end, the execution of a node is divided into epochs. Each epoch is further divided into phases. Each phase consists of a certain number of time-slots that depends on the epoch number. Intuitively, an epoch corresponds to one estimate value for $T_{\text {est }}$. Different phases within an epoch correspond to different estimate values for $\Delta_{e s t}$.

We now describe the specifics of our approach. Epoch $i$, for $i=1,2,3, \ldots$, consists of $i+1$ phases with each phase consisting of $2^{i}$ time-slots. In phase $j$ of epoch $i$, where $1 \leq j \leq i+1$, we set the estimate value for $\Delta_{\text {est }}$ to $2^{j}$. In other words, during phase $j$ of epoch $i$, we basically execute an instance of Algorithm 3 with $T_{e s t}$ set to $2^{i}$ and $\Delta_{e s t}$ set to $2^{j}$. A formal description of the algorithm is shown in Algorithm 4.

In order to show that Algorithm 4 guarantees that neighbor discovery completes with high probability within a certain number of time-slots, it is sufficient to show that there exists an epoch containing a phase such that: (i) nodes use a sufficiently large value as an estimate for maximum node degree during the phase, and (ii) the phase for all nodes overlaps for a sufficiently long time so as to allow all links to be discovered.

Let $\Delta_{0}$ denote the smallest power of two greater than or equal to $\Delta$. Further, let $M=\frac{16 \max \left(S, \Delta_{0}\right)}{\rho} \ln \left(\frac{N^{2}}{\epsilon}\right)$. Note that the expression for $M$ is derived from the number of time-slots needed to achieve the success probability of at least $1-\epsilon$ when nodes use $\Delta_{0}$ as their estimate for the maximum node degree. Given a node $u$, we refer to the phase numbered $\log \Delta_{0}$ of the epoch numbered $\lceil\log (M+\Theta)\rceil$ as the potent phase of node $u$. (We call it potent because we show that neighbor discovery is guaranteed to complete with desired probability in this phase.) In its potent phase, each node executes an instance of Algorithm 3 with $\Delta_{e s t}$ set to $\Delta_{0}$ for at least $M+\Theta$ time-slots. Let $T_{b}$ denote the latest time at which some node begins executing its potent phase. Likewise, let $T_{e}$ denote the earliest time at which some node ends executing its potent phase. 
Note that the time difference between when two nodes start the same phase is same as the slack between the two nodes (the time difference between when two nodes start the neighbor discovery algorithm). Since the maximum slack between any two nodes is $\Theta$, clearly, $T_{e}-T_{b} \geq M$. This implies that, during the time period from $T_{b}$ to $T_{e}$, the following holds: (i) the potent phases of all nodes mutually overlap in time, and (ii) the time-period contains at least $M$ time-slots from the potent phase of every node. We refer to the period of time from $T_{b}$ to $T_{e}$ as the potent period. During the potent period, each node uses the same estimate for the upper bound on maximum node degree, namely $\Delta_{0}$. Using an analysis similar to that for the previous algorithm, it can be shown that a single time-slot during the potent period covers a given link with probability at least $\frac{\rho}{16 \max \left(S, \Delta_{0}\right)}$. This in turn implies that the probability that neighbor discovery completes successfully during the potent period is at least $1-\epsilon$.

Note that epoch $i$ contains $(i+1) \cdot 2^{i}$ time-slots. Thus, the total number of time-slots contained in epochs 1 through $i$ is given by $i \cdot 2^{i+1}$ (proof is given in the appendix). As a result, we have:

Theorem 4. Algorithm 4 guarantees that each node discovers all its neighbors on all channels within $O((M+\Theta) \log (M+\Theta))$ time-slots of $T_{s}$ with probability at least $1-\epsilon$, where $M=\frac{16 \max \left(S, \Delta_{0}\right)}{\rho} \ln \left(\frac{N^{2}}{\epsilon}\right)$.

\section{Neighbor Discovery in Asynchronous System}

We first describe an algorithm that assumes that nodes know a good upper bound on maximum node degree. We then describe a more general algorithm in which no such knowledge is assumed. The second algorithm, however, requires a tighter bound on the clock drift rate than the first algorithm. Both algorithms are derived from their synchronous counterparts in which different nodes can start at different times.

The main idea is to construct a framework that allows each node to essentially use the slotted-time abstraction (for its local time) and run a modified version of either Algorithm 3 or Algorithm 4. However, unlike in a synchronous system, slots of different nodes need not be aligned. They may not even be of the same length. In fact, the slot length for the same node may also vary with time due to unpredictable drift rate of its clock. (Note that any node will use its own clock to measure the passage of time.) However, if the maximum drift rate of any clock is bounded, we can still establish good theoretical bounds on the running time of the asynchronous algorithms similar to that for its synchronous counterparts. 


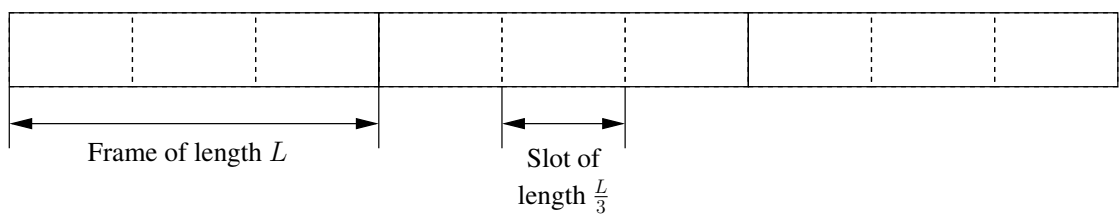

Figure 1: Execution of a node with respect to its local clock. All frames are of length $L$. All slots are of length $\frac{L}{3}$.

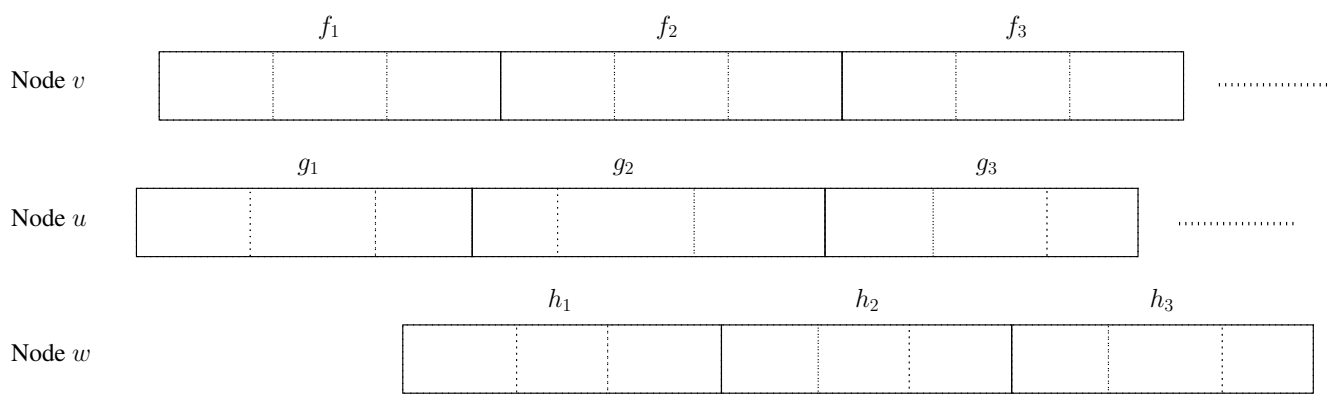

Figure 2: Execution of the network with respect to real time. Frames may be of different lengths (even within the same node). Slots may be of different lengths (even within the same frame).

\subsection{Knowledge of Good Upper Bound on Maximum Node Degree}

We adapt Algorithm 3 that works for the synchronous case to work for the asynchronous case. Let $\Delta_{e s t}$ denote an upper bound on the maximum node degree as known to all nodes. Our algorithm makes the following assumption about the maximum drift rate of the clock of any node $\delta$ :

Assumption 1. The maximum drift rate of the clock of any node is bounded by $\frac{1}{7}$ seconds/second.

The offset or skew between clocks of any two nodes may be arbitrarily large and, in fact, may grow with time. We now describe how to extend Algorithm 3 to solve the neighbor discovery algorithm in an asynchronous system.

Each node divides its time into equal-sized frames. Frames of different nodes are not required to be synchronized and may, in fact, be misaligned. A node measures the duration of a frame using its local clock. The length of a frame as measured by a node using its local clock is same for all nodes, say L. Note that, because of the clock drift, duration of a frame, when projected on real time, may be different from $L$ - shorter than $L$ in case of positive drift and longer than $L$ in case of negative drift. Specifically, it can be verified that the length of a frame in real time lies in the range:

$$
\frac{L}{1+\delta} \leq \text { frame length in real-time } \leq \frac{L}{1-\delta}
$$




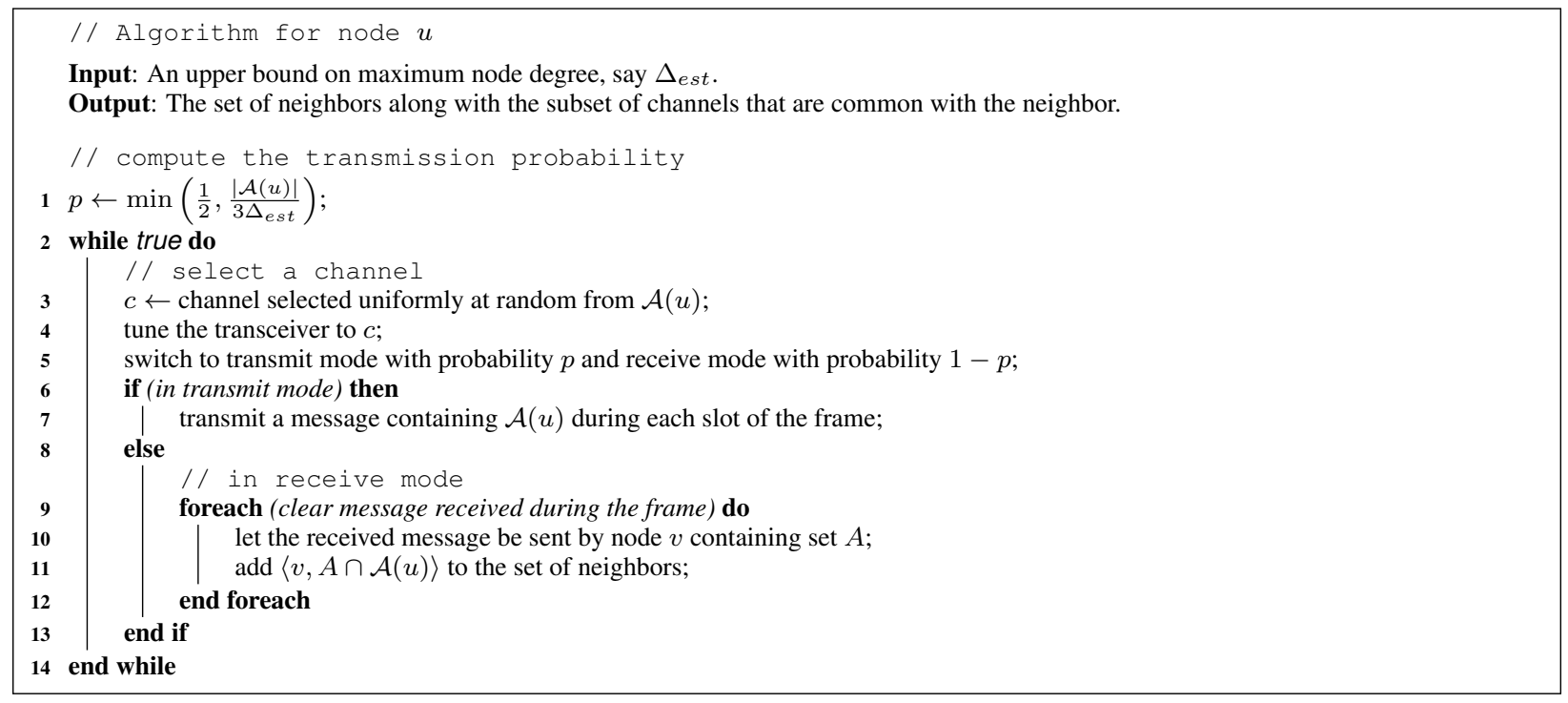

Algorithm 5: Neighbor discovery algorithm for an asynchronous system with a bound on clock drift rate and knowledge of upper bound on maximum node degree.

A node further divides each frame into three equal-sized slots-equal with respect to its local clock. Therefore the duration of a slot as measured by a node using its local clock is $\frac{L}{3}$. At the beginning of each frame, a node $u$ randomly selects a channel from its available channel set, say $c$. It then transmits on $c$ during each slot of the frame with probability $\min \left(\frac{1}{2}, \frac{|\mathcal{A}(u)|}{3 \Delta_{e s t}}\right)$ and listens on $c$ during the entire frame with the remaining probability. In the former case, $u$ transmits the same message during each slot of the frame. In the latter case, $u$ listens for any clear messages it may receive during any part of the frame. The partition of a frame into slots is not important for a receiving node; they are only used by a transmitting node. Also, a node may receive multiple clear messages while listening during a single frame. The execution of a node with respect to its local clock is shown in Fig. 1. The execution of the network with respect to common real time is shown in Fig. 2.

A formal description of the algorithm is shown in Algorithm 5. We now analyze the running time of the algorithm. In our analysis, unless otherwise stated, time refers to real-time. Of course, nodes do not have access to the real-time. We next define two notions we use in the analysis of the our algorithm.

Definition 1 (aligned pair). We say that a pair of frames $\langle f, g\rangle$ is aligned, where $f$ and $g$ are frames of two different nodes, if at least one slot of $f$ lies completely within $g$.

For example, in the execution shown in Fig. 2, pairs $\left\langle f_{1}, g_{1}\right\rangle$ and $\left\langle f_{2}, h_{1}\right\rangle$ are aligned whereas the pair $\left\langle f_{1}, h_{1}\right\rangle$ is not. Intuitively, a aligned pair of frames in the asynchronous case is analogous to a pair of 
overlapping time-slots in the synchronous case. A aligned pair of frames allows the two nodes to exchange a message under certain conditions (explained later).

Definition 2 (overlapping frames). For a frame $f$ and a node $u$, let overlap $(f, u)$ denote the subset of frames of $u$ that overlap in real-time with $f$. Further, let overlap-all $(f)$ denote the subset of all frames that overlap in real-time with $f$.

For example, in the execution shown in Fig. 2, overlap $\left(g_{2}, v\right)=\left\{f_{1}, f_{2}\right\}$ and overlap-all $\left(g_{2}\right)=$ $\left\{f_{1}, f_{2}, g_{2}, h_{1}, h_{2}\right\}$.

Consider the link from node $v$ to node $u$ on channel $c$. Also, consider frames $f$ of $v$ and $g$ of $u$ such that the pair $\langle f, g\rangle$ is aligned. We extend the notion of a link covered by a time-slot (defined in Section 3.1.1) to a link covered by an aligned pair of frames. Specifically, the pair of aligned frames $\langle f, g\rangle$ covers the link $(v, u)$ on channel $c$ if: (i) $v$ transmits on $c$ during $f$, (ii) $u$ listens on $c$ during $g$, and (iii) no other neighbor of $u$, say $w$, transmits on $c$ during any frame in $\operatorname{overlap}(g, w)$. Also, $\langle f, g\rangle$ covers $(v, u)$ if $\langle f, g\rangle$ covers $(v, u)$ on some channel $c \in \operatorname{span}(v, u)$. The three conditions collectively ensure that $u$ receives a clear message from $v$ (on $c$ during $g$ ) provided $\langle f, g\rangle$ is aligned.

One of the arguments we commonly use in our analysis is: " $x$ adjacent slots of a node cannot strictly contain $y$ adjacent slots of another node" for certain specific values of $x$ and $y$ with $x<y$. To prove this, we argue that, for the statement to be false, the following inequality must hold:

$$
\frac{x L}{3(1-\delta)}>\frac{y L}{3(1+\delta)}
$$

and then show that it contradicts Assumption 1. The above inequality must hold for the statement to be false because the left hand side denotes the largest possible length of the time interval containing $x$ adjacent slots, and the right hand side denotes the smallest possible length of the time interval containing $y$ adjacent slots.

Lemma 5. A frame of a node overlaps with at most three frames of any other node. Formally,

$$
\forall f, u::|\operatorname{overlap}(f, u)| \leq 3
$$

Proof. Assume, in order to obtain a contradiction, that a frame of some node, say $f$, overlaps with at least four frames of another node. This implies that there are at least two consecutive frames that are strictly 
contained within $f$. From (10), a frame of a node can strictly contain two frames of another node only if the following condition holds:

$$
\frac{L}{1-\delta}>\frac{2 L}{1+\delta} \quad \Longrightarrow \quad \delta>\frac{1}{3}
$$

This contradicts Assumption 1.

We now show that a aligned pair of frames allows a link to be discovered with a significant probability. We have:

Lemma 6. If $\langle f, g\rangle$ is aligned, then $\langle f, g\rangle$ covers $(v, u)$ with probability at least $\frac{\rho}{8 \max \left(2 S, 3 \Delta_{e s t}\right)}$.

Proof. Analogous to $A(\tau, c), B(\tau, c)$ and $C(\tau, c)$ defined in Section 3.1.1, let $\hat{A}(f, g, c), \hat{B}(f, g, c), \hat{C}(f, g, c)$ denote the three events corresponding to the three conditions (i), (ii) and (iii), respectively, required for coverage. Note that the three events are mutually independent. As before, we compute the probability of occurrence of the three events separately.

Computing the probability of occurrence of $\hat{A}(f, g, c)$ : We have:

$\operatorname{Pr}\{\hat{A}(f, g, c)\}=(v$ selects $c$ at the beginning of $f) \wedge(v$ chooses to transmit during $f)$

$$
\begin{aligned}
& =\frac{1}{|\mathcal{A}(v)|} \times \min \left(\frac{1}{2}, \frac{|\mathcal{A}(v)|}{3 \Delta_{e s t}}\right)=\min \left(\frac{1}{2|\mathcal{A}(v)|}, \frac{1}{3 \Delta_{e s t}}\right) \\
& \geq \frac{1}{\max \left(2 S, 3 \Delta_{e s t}\right)}
\end{aligned}
$$

Computing the probability of occurrence of $\hat{B}(f, g, c)$ : We have:

$\operatorname{Pr}\{\hat{B}(f, g, c)\}=(u$ selects $c$ at the beginning of $g) \wedge(u$ chooses to listen during $g)$

$$
\begin{aligned}
& =\frac{1}{|\mathcal{A}(u)|} \times\left\{1-\min \left(\frac{1}{2}, \frac{|\mathcal{A}(u)|}{3 \Delta_{\text {est }}}\right)\right\} \\
& \{\min (x, y) \leq x\} \\
& \geq \frac{1}{|\mathcal{A}(u)|} \times\left(1-\frac{1}{2}\right)=\frac{1}{2|\mathcal{A}(u)|}
\end{aligned}
$$


Computing the probability of occurrence of $\hat{C}(f, g, c)$ : Let $\mathcal{N}(u, c)$ denote the set of neighbors of $u$ on c. Note that, if $\mathcal{N}(u, c)$ only contains $v$, then $\operatorname{Pr}\{\hat{C}(f, g, c)\}=1$. Otherwise, we have:

$$
\begin{aligned}
& \operatorname{Pr}\{\hat{C}(f, g, c)\} \\
& =\prod_{\substack{w \in \mathcal{N}(u, c) \\
w \neq v}} \operatorname{Pr}\left(\begin{array}{c}
w \text { does not transmit on } c \text { during any frame } \\
\text { in } \operatorname{overlap}(g, w)
\end{array}\right)
\end{aligned}
$$

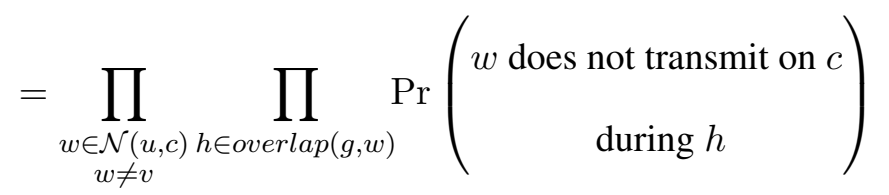

$$
\begin{aligned}
& =\prod_{\substack{w \in \mathcal{N}(u, c) \\
w \neq v}} \prod_{h \in \text { overlap }(g, w)}\{1-\operatorname{Pr}(w \text { transmits on } c \text { during } h)\} \\
& \geq \prod_{\substack{w \in \mathcal{N}(u, c) \\
w \neq v}} \prod_{h \in \operatorname{overlap}(g, w)}\left\{1-\frac{1}{|\mathcal{A}(w)|} \times \min \left(\frac{1}{2}, \frac{|\mathcal{A}(w)|}{3 \Delta_{\text {est }}}\right)\right\} \\
& =\prod_{\substack{w \in \mathcal{N}(u, c) \\
w \neq v}} \prod_{h \in \operatorname{overlap}(g, w)}\left\{1-\min \left(\frac{1}{2|\mathcal{A}(w)|}, \frac{1}{3 \Delta_{\text {est }}}\right)\right\} \\
& \{\min (x, y) \leq y\} \\
& \geq \prod_{\substack{w \in \mathcal{N}(u, c) \\
w \neq v}} \prod_{h \in \operatorname{overlap}(g, w)}\left(1-\frac{1}{3 \Delta_{\text {est }}}\right) \\
& \{\text { using Lemma 5, } \mid \text { overlap }(g, w) \mid \leq 3\} \\
& \geq \prod_{\substack{w \in \mathcal{N}(u, c) \\
w \neq v}}\left(1-\frac{1}{3 \Delta_{e s t}}\right)^{3}=\left(1-\frac{1}{3 \Delta_{e s t}}\right)^{3(|\mathcal{N}(u, c)|-1)} \\
& \left\{|\mathcal{N}(u, c)|-1=\Delta(u, c)-1 \leq \Delta_{e s t}\right\} \\
& \geq\left(1-\frac{1}{3 \Delta_{e s t}}\right)^{3 \Delta_{e s t}} \\
& \left\{\forall x \geq 2,\left(1-\frac{1}{x}\right)^{x} \text { is a monotonically increasing function of } x \text { and thus } \geq \frac{1}{4}\right\} \\
& \geq \frac{1}{4}
\end{aligned}
$$

Finally, using a derivation similar to that for (6), it can be shown that $\langle f, g\rangle$ covers $(v, u)$ with probability at least $\frac{\rho}{8 \max \left(2 S, 3 \Delta_{\text {est }}\right)}$. 
For a frame $f$, we use node $(f)$ to denote the node to which $f$ belongs. For example, as per the execution show in Fig. 2, node $\left(f_{1}\right)=v$ and node $\left(h_{1}\right)=w$. We now define a precedence relation between pairs of frames referred to as frame-pairs for short.

Definition 3 (precedence relation). For frame-pairs $\langle f, g\rangle$ and $\langle p, q\rangle$, we say that $\langle f, g\rangle$ precedes $\langle p, q\rangle$, denoted $\langle f, g\rangle \sqsubset\langle p, q\rangle$, if $(i)$ node $(f)=\operatorname{node}(p)$, (ii) node $(g)=$ node $(q)$, (iii) the start time of $f$ is before that of $p$, and (iv) the start time of $g$ is before that of $q$.

For example, as per the execution shown in Fig. 2, $\left\langle f_{1}, g_{1}\right\rangle \sqsubset\left\langle f_{2}, g_{2}\right\rangle$ and $\left\langle f_{1}, g_{1}\right\rangle \sqsubset\left\langle f_{3}, g_{2}\right\rangle$ but $\left\langle f_{1}, g_{1}\right\rangle \not \subset\left\langle f_{1}, g_{2}\right\rangle$. Intuitively, if two frame-pairs have a precedence relation between them, then the coverage provided by the two frame-pairs can be treated as independent events under certain conditions as captured by the next notion, thereby making the analysis tractable.

Definition 4 (admissible sequence). A sequence $\sigma$ of $M$ frame-pairs $\left\{\left\langle f_{i_{k}}, g_{j_{k}}\right\rangle\right\}_{1 \leq k \leq M}$ is admissible with respect to the link $(v, u)$ if it satisfies the following conditions:

1. $\forall i: 1 \leq k \leq M: \operatorname{node}\left(f_{i_{k}}\right)=v$ and $\operatorname{node}\left(g_{j_{k}}\right)=u$,

2. $\forall i: 1 \leq k<M:\left\langle f_{i_{k}}, g_{j_{k}}\right\rangle \sqsubset\left\langle f_{i_{k+1}}, g_{j_{k+1}}\right\rangle$,

3. $\forall i: 1 \leq k \leq M:\left\langle f_{i_{k}}, g_{j_{k}}\right\rangle$ is aligned, and

4. $\forall i: 1 \leq k<M$ : overlap-all $\left(g_{i_{k}}\right) \cap$ overlap-all $\left(g_{i_{k+1}}\right)=\emptyset$.

An admissible sequence of frame-pairs in the asynchronous case is essentially analogous to a sequence of time-slots in the synchronous case. If a single frame-pair guarantees that an event occurs with a certain probability, then a "sufficiently long" admissible sequence of frame-pairs can be used to increase that probability to any desired value. Table 1 shows the analogous concepts between synchronous and asynchronous systems. For a sequence $\sigma$ of frame-pairs that is admissible with respect to $(v, u)$, we say that $\sigma$ covers $(v, u)$ if some frame-pair in the sequence covers $(v, u)$.

Lemma 7. Let $\sigma$ be a sequence of $\frac{8 \max \left(2 S, 3 \Delta_{e s t}\right)}{\rho} \ln \left(\frac{N^{2}}{\epsilon}\right)$ frame-pairs such that $\sigma$ is admissible with respect to $(v, u)$. Then the probability that $\sigma$ does not cover $(v, u)$ is at most $\frac{\epsilon}{N^{2}}$.

Proof. For convenience, let $M=\frac{8 \max \left(2 S, 3 \Delta_{e s t}\right)}{\rho} \ln \left(\frac{N^{2}}{\epsilon}\right)$. Let $E_{k}$ with $1 \leq k \leq M$ denote the event that $\left\langle f_{i_{k}}, g_{j_{k}}\right\rangle$ covers $(v, u)$ on some channel. Note that the occurrence of the event $E_{k}$ depends only on 
Table 1: Analogous terms for synchronous and asynchronous systems.

\begin{tabular}{|l|l|}
\hline \multicolumn{1}{|c|}{ Synchronous System } & \multicolumn{1}{c|}{ Asynchronous System } \\
\hline slot & frame \\
\hline slot pair & frame-pair \\
\hline overlapping slot pair & aligned frame-pair \\
\hline sequence of distinct overlapping slot pairs & admissible sequence of frame-pairs \\
\hline
\end{tabular}

frames in overlap-all $\left(g_{j_{k}}\right)$. Since $\sigma$ is an admissible sequence, for all $x$ and $y$, with $1 \leq x<y \leq M$, overlap-all $\left(g_{j_{x}}\right) \cap$ overlap-all $\left(g_{j_{y}}\right)=\emptyset$. In other words, the set of frames that overlap with the frame $g_{j_{x}}$ are distinct from the set of frames that intersect the frame $g_{j_{y}}$. Since, for each frame, a node chooses its action randomly, events $E_{k}$ s are mutually independent of each other. Using a derivation similar to that of (7), it can be shown that $\sigma$ does not cover $(v, u)$ with probability at most $\frac{\epsilon}{N^{2}}$.

We next show that any execution of the network must contain a "sufficiently long" sequence of admissible frame-pairs. In the remainder of this section, let $T_{s}$ denote the time by which all nodes have initiated the neighbor discovery algorithm, Algorithm 5. To show the existence of a "sufficiently long" admissible sequence, we first show that, for any instant of time $T$ after $T_{s}$ and, for any pair of neighboring nodes, there is an aligned pair of frames after but "close" to $T$.

Lemma 8. Consider a link from node $v$ to node $u$ and some instant of time $T$ with $T \geq T_{s}$. Let $f_{i}$ (respectively, $g_{i}$ ) with $i \geq 1$ denote the $i^{\text {th }}$ full frame of node $v$ (respectively, node $u$ ) after $T$. Then some frame in $\left\{f_{1}, f_{2}\right\}$ is aligned with some frame in $\left\{g_{1}, g_{2}\right\}$.

The proof of the above lemma is somewhat technical and has been moved to the appendix (see Section B). We now show that existence of a "sufficiently long" admissible sequence in any execution of Algorithm 5.

Lemma 9. Consider a link from node $v$ to node $u$. Further, consider an execution of the network after $T_{s}$ that contains at least $M$ full frames of $u$ as well as $v$. Then the execution contains a sequence of at least $\frac{M}{6}$ frame-pairs such that the sequence is admissible with respect to $(v, u)$.

Proof. The proof is by construction. The construction is in two steps. In the first step, we construct a sequence of frame-pairs $\gamma$ that is "almost" admissible in the sense that it satisfies the first three properties of 
an admissible sequence but may not satisfy the fourth property. We show that $\gamma$ contains at least $\frac{M}{2}$ framepairs. In the second step, using $\gamma$, we construct a sequence of frame-pairs $\sigma$ that satisfies all four properties of an admissible sequence. We also show that $\sigma$ contains at least $\frac{M}{6}$ frame-pairs.

Constructing $\gamma$ : To obtain the first frame-pair $\left\langle f_{i_{1}}, g_{i_{1}}\right\rangle$ that is aligned, we apply Lemma 8 to $T_{s}$. Now, assume that we have already selected $k$ frame-pairs satisfying the first three properties of an admissible sequence. Let the $k^{t h}$ frame-pair be denoted by $\left\langle f_{i_{k}}, g_{i_{k}}\right\rangle$. To select the next frame-pair, let $T_{k}$ be defined as the earlier of the end times of frames $f_{i_{k}}$ and $g_{i_{k}}$. To obtain the next frame-pair that is aligned, we apply Lemma 8 to $T_{k}$. Let the frame-pair be denoted by $\left\langle f_{i_{k+1}}, g_{i_{k+1}}\right\rangle$. Clearly, the extended sequence (consisting of $k+1$ aligned frame-pairs) satisfies the first and third properties of an admissible sequence. The second property holds because the start times of both $f_{i_{k}}$ and $g_{i_{k}}$ are before $T_{k}$, whereas the start times of both $f_{i_{k+1}}$ and $g_{i_{k+1}}$ are after $T_{k}$. We repeatedly select aligned frame-pairs using Lemma 8 until we run out of frames of either $u$ or $v$. We now establish a lower bound on the length of $\gamma$. Note that, when selecting the $(k+1)^{s t}$ pair, the first full frame of node $v$ after $T_{k}$ (namely frame $f_{1}$ in the statement of Lemma 8) is adjacent to the frame $f_{i_{k}}$. This follows from the definition of $T_{k}$. This, in turn, implies that the frame $f_{i_{k+1}}$ obtained using Lemma 8 is within a distance of two of the frame $f_{i_{k}}$. Likewise, the frame $g_{i_{k+1}}$ is within a distance of two of the frame $g_{i_{k}}$. As a result, $\gamma$ contains at least $\frac{M}{2}$ frame-pairs.

Constructing $\sigma$ : To construct a sequence $\sigma$ that also satisfies the fourth property of an admissible sequence, we choose every third frame-pair of $\gamma$ starting with the first frame-pair $\left\langle f_{i_{1}}, g_{i_{1}}\right\rangle$. Clearly $\sigma$ also satisfies the first three properties of an admissible sequence (since it is a subsequence of $\gamma$ ). Let the $k^{t h}$ frame-pair of $\sigma$ be denoted by $\left\langle f_{j_{k}}, g_{j_{k}}\right\rangle$. To prove that $\sigma$ satisfies the fourth property as well, assume, by the way of contradiction, that some frame, say $h$, overlaps with two consecutive frames $g_{j_{k}}$ and $g_{j_{k+1}}$ for some $k$. Note that, since we selected only every third frame-pair of $\gamma$ to construct $\sigma$, there are at least two other frames of $u$ between $g_{j_{k}}$ and $g_{j_{k+1}}$. This implies that $h$ overlaps with at least four frames of $u$, which contradicts Lemma 5. This establishes that the length of $\sigma$ is at least $\frac{M}{6}$.

Finally, we have the main result.

Theorem 10. Let $T_{s}$ be the time by which all nodes have initiated neighbor discovery. Also, let $T_{f}$ be the earliest time by which each node has executed at least $\frac{48 \max \left(2 S, 3 \Delta_{e s t}\right)}{\rho} \ln \left(\frac{N^{2}}{\epsilon}\right)$ full frames since $T_{s}$. Then 
Algorithm 5 ensures that each node discovers all its neighbors on all channels with probability at least $1-\epsilon$ by time $T_{f}$.

Proof. Consider a link $(v, u)$. From Lemma 9, the execution from $T_{s}$ to $T_{f}$ contains a sequence of at least $\frac{8 \max \left(2 S, 3 \Delta_{e s t}\right)}{\rho} \ln \left(\frac{N^{2}}{\epsilon}\right)$ frame-pairs such that the sequence is admissible with respect to $(v, u)$. From Lemma 7, the probability that $(v, u)$ is not covered by $T_{f}$ is at most $\frac{\epsilon}{N^{2}}$. This implies that the probability that some link in the network is not covered by $T_{f}$ is at most $\epsilon$.

We now bound the length of the interval $T_{f}-T_{s}$.

Theorem 11. Let $T_{s}$ and $T_{f}$ be as defined in Theorem 10. Then the length of the interval $T_{f}-T_{s}$ is upper bounded by $\left\{\frac{48 \max \left(2 S, 3 \Delta_{e s t}\right)}{\rho} \ln \left(\frac{N^{2}}{\epsilon}\right)+1\right\}\left(\frac{L}{1-\delta}\right)$.

Proof. By our choice of $T_{f}$, there exists some node such that $T_{f}-T_{s}$ contains exactly $\frac{48 \max \left(2 S, 3 \Delta_{e s t}\right)}{\rho} \ln \left(\frac{N^{2}}{\epsilon}\right)$ full frames of that node. The first frame of that node in the execution may be partial frame. From (10), the length of each frame is upper bounded by $\frac{L}{1-\delta}$. Combining the three facts, we obtain the result.

\subsection{No Knowledge of Maximum Node Degree}

As in the synchronous case, the main idea is to repeatedly execute instances of Algorithm 5 for different values of $\Delta_{\text {est }}$. Again, the main challenge is to determine how long to run each instance of Algorithm 5 in order to achieve arbitrarily high success probability without knowing any of the system parameters. Let $T_{\text {est }}$ denote the amount of time (in terms of number of frames) for which an instance of Algorithm 5 is run. We need to simultaneously estimate both $\Delta_{e s t}$ and $T_{\text {est }}$ so as to guarantee that neighbor discovery completes with arbitrarily high probability. As in the synchronous case, the execution of a node is divided into epochs. Each epoch is further divided into phases. Each phase consists of a certain number of frames that depends on the epoch number. Intuitively, an epoch corresponds to one estimate value for $T_{\text {est }}$. Different phases within an epoch correspond to different estimate values for $\Delta_{e s t}$. In particular, epoch $i$, for $i=1,2,3, \ldots$, consists of $i+1$ phases with each phase consisting of $2^{i}$ frames. In phase $j$ of epoch $i$, where $1 \leq j \leq i+1$, we set the estimate value for $\Delta_{e s t}$ to $2^{j}$. In other words, during phase $j$ of epoch $i$, we basically execute an instance of Algorithm 5 with $T_{e s t}$ set to $2^{i}$ and $\Delta_{e s t}$ set to $2^{j}$. A formal description of the algorithm is given in Algorithm 6. 


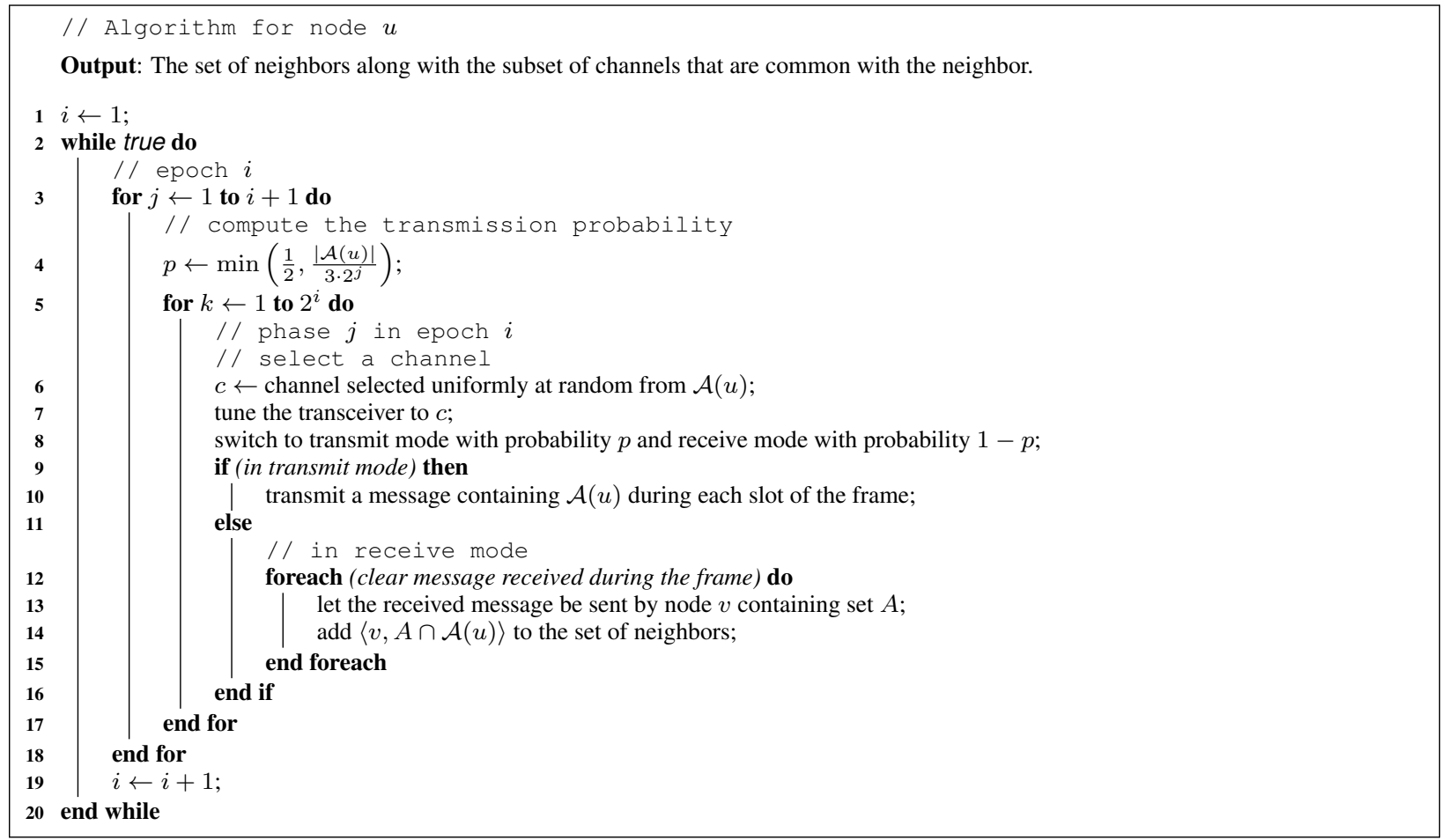

Algorithm 6: Neighbor discovery algorithm for an asynchronous system with a bound on clock drift rate (no other knowledge assumed).

The time-complexity analysis of Algorithm 6 is more involved because of the asynchrony. Unlike in a synchronous system, the time difference between when two nodes start the same phase may grow with time due to clock drift. If the clock drift rate is too large, then two nodes may start their critical phases so long apart that there may not be enough number of overlapping frames to guarantee completion of neighbor discovery with sufficiently high probability.

We first define some additional notation we need for the algorithm analysis. Let $\Lambda(i, j)$ denote the phase $j$ of the epoch $i$. Let $F_{i, j}$ denote the number of frames that a node in the execution until (but not including) $\Lambda(i, j)$. We have:

$$
\begin{aligned}
F_{i, j}= & \text { (number of frames contained in the first } i-1 \text { epochs })+ \\
& \text { (number of frames contained in the } \left.i^{\text {th }} \text { epoch until } \Lambda(i, j)\right) \\
& \left\{\text { using }(\mathrm{C}) \text { in the appendix and the fact that, for each } j,|\Lambda(i, j)|=2^{i}\right\} \\
= & (i-1) \cdot 2^{i}+(j-1) \cdot 2^{i}=(i+j-2) \cdot 2^{i}
\end{aligned}
$$


Let $T_{b}^{u}(i, j)$ denote the time at which node $u$ begins executing $\Lambda(i, j)$. Likewise, let $T_{e}^{u}(i, j)$ denote the time at which node $u$ ends executing $\Lambda(i, j)$. Let $T_{b}^{\max }(i, j)$ denote the latest time at which some node starts executing $\Lambda(i, j)$. Likewise, let $T_{e}^{\min }(i, j)$ denote the earliest time at which some node starts executing $\Lambda(i, j)$. Symbols $T_{s}, \Theta$ and $L$ have the same meaning as before. Note that, in real-time, the minimum and maximum lengths of a frame are given by $\frac{L}{1+\delta}$ and $\frac{L}{1-\delta}$, respectively. Thus, we have:

$$
\begin{aligned}
& T_{b}^{\max }(i, j)=\max \left\{T_{b}^{u}(i, j)\right\} \leq T_{s}+F_{i, j}\left(\frac{L}{1-\delta}\right) \\
& T_{e}^{\min }(i, j)=\min \left\{T_{e}^{u}(i, j)\right\} \geq\left(T_{s}-\Theta\right)+\left(F_{i, j}+2^{i}\right)\left(\frac{L}{1+\delta}\right)
\end{aligned}
$$

Suppose $i$ and $j$ are such that $T_{e}^{\min }(i, j)<T_{b}^{\max }(i, j)$. Note that, during the time-period from $T_{b}^{\max }(i, j)$ to $T_{e}^{\min }(i, j)$, all nodes will use the same estimate for maximum node degree. Recall that $\Delta_{0}$ denotes the smallest power of two greater than or equal to $\delta$. Let $M=\frac{48 \max \left(2 S, 3 \Delta_{0}\right)}{\rho} \ln \left(\frac{N^{2}}{\epsilon}\right)+1$. From Theorem 10 , if the time period from $T_{b}^{\max }(i, j)$ to $T_{e}^{\min }(i, j)$ is such that: (a) the time period contains at least $M$ full frames and (b) each node uses an estimate value of $\Delta_{0}$ during the time period, then the neighbor discovery is guaranteed to complete with probability at least $1-\epsilon$. Let $i_{0}=\left\lceil\log \left(6 M+\frac{3 \Theta}{L}\right)\right\rceil$ and $j_{0}=\lceil\log \Delta\rceil(=$ $\left.\log \Delta_{0}\right)$. Note that, by definition, during $\Lambda\left(i_{0}, j_{0}\right)$, each node uses $\Delta_{0}$ as the estimate for maximum node degree. It suffices to prove that the time period from $T_{s} i_{0} j_{0}$ to $T_{f} i_{0} j_{0}$ contains at least $M$ frames. To that end, we make the following assumption about the clock drift rate:

Assumption 2. Let $D=48\left\{\log \left(\frac{N S}{\epsilon}\right)+\log \left(1+\frac{\Theta}{L}\right)+5\right\}$. The maximum drift rate of the clock of any node is bounded by $\frac{1}{D}$ seconds/second.

We first prove the following lemma:

Lemma 12. If Assumption 2 holds, then $i_{0} \cdot \delta \leq \frac{1}{16}$.

Proof. We have:

$$
\begin{aligned}
M & =\frac{48 \max \left(2 S, 3 \Delta_{0}\right)}{\rho} \ln \left(\frac{N^{2}}{\epsilon}\right)+1 \\
& \leq\left(\frac{48 \cdot(2 S) \cdot(6 N)}{\frac{1}{S}}\right) \cdot\left(\frac{N^{2}}{\epsilon}\right)+1=\frac{576 \cdot S^{2} \cdot N^{3}}{\epsilon}+1
\end{aligned}
$$


This implies that:

$$
\begin{aligned}
\log M & \leq \log 576+3 \log \left(\frac{N S}{\epsilon}\right)+1 \\
& \leq 3 \log \left(\frac{N S}{\epsilon}\right)+11
\end{aligned}
$$

Now, we have:

$$
\begin{aligned}
i_{0} \cdot \delta \leq & \left\{\log \left(6 M+\frac{3 \Theta}{L}\right)+1\right\} \cdot \delta \\
\leq & \left\{\log 6+\log \left(M+\frac{\Theta}{L}+1\right)+1\right\} \cdot \delta \\
& \{\text { for all } x, y \text { with } x \geq 1 \text { and } y \geq 1, \log (x+y) \leq \log (x y) \leq \log x+\log y\} \\
\leq & \left\{\log (M)+\log \left(1+\frac{\Theta}{L}\right)+4\right\} \cdot \delta \\
& \{\text { using }(17)\} \\
\leq & \left\{3 \log \left(\frac{N S}{\epsilon}\right)+11+\log \left(1+\frac{\Theta}{L}\right)+4\right\} \cdot \delta \leq 3 \cdot\left\{\log \left(\frac{N S}{\epsilon}\right)+\log \left(1+\frac{\Theta}{L}\right)+5\right\} \cdot \delta \\
& \{\text { using definition of } D\} \\
\leq & 3 \cdot \frac{D}{48} \cdot \delta \\
& \{\text { using Assumption } 2\} \\
\leq & 3 \cdot \frac{D}{48} \cdot \frac{1}{D}=\frac{1}{16}
\end{aligned}
$$

This establishes the lemma.

Now, substituting $i=i_{0}$ and $j=j_{0}$ in (15) and (16) and subtracting (15) from (16), we obtain:

$$
\begin{aligned}
& T_{e}^{\min }\left(i_{0}, j_{0}\right)-T_{b}^{\max }\left(i_{0}, j_{0}\right) \\
& \geq\left(F_{i_{0}, j_{0}}+2^{i_{0}}\right)\left(\frac{L}{1+\delta}\right)-F_{i_{0}, j_{0}}\left(\frac{L}{1-\delta}\right)-\Theta \\
& \quad\{\text { rearranging terms }\} \\
& =2^{i_{0}}\left(\frac{L}{1+\delta}\right)-F_{i_{0}, j_{0}}\left(\frac{2 \delta L}{1-\delta^{2}}\right)-\Theta \\
& \quad\{\text { using (14) }
\end{aligned}
$$




$$
\begin{aligned}
= & 2^{i_{0}}\left(\frac{L}{1+\delta}\right)-\left(i_{0}+j_{0}-2\right) \cdot 2^{i_{0}}\left(\frac{2 \delta L}{1-\delta^{2}}\right)-\Theta \\
\geq & 2^{i_{0}}\left(\frac{L}{1+\delta}\right)\left\{1-\left(i_{0}+j_{0}-2\right)\left(\frac{2 \delta}{1-\delta}\right)\right\}-\Theta \\
& \left\{i_{0}+j_{0}-2 \leq 2 i_{0}\right\} \\
\geq & 2^{i_{0}}\left(\frac{L}{1+\delta}\right)\left(1-\frac{\left(2 i_{0}\right)(2 \delta)}{1-\delta}\right)-\Theta \\
& \left\{\delta \leq \frac{1}{2}\right\} \\
\geq & 2^{i_{0}}\left(\frac{2 L}{3}\right)\left(1-8 \cdot i_{0} \cdot \delta\right)-\Theta \\
& \{\text { using Lemma } 12\} \\
\geq & 2^{i_{0}}\left(\frac{L}{3}\right)-\Theta \geq\left(6 M+\frac{3 \Theta}{L}\right)\left(\frac{L}{3}\right)-\Theta=2 M L
\end{aligned}
$$

We now prove the main result. Since the maximum length of a frame in real-time is $\frac{L}{1-\delta}$ and $\delta \leq \frac{1}{2}$, it follows that the time-period from $T_{b}^{\max }\left(i_{0}, j_{0}\right)$ to $T_{b}^{\max }\left(i_{0}, j_{0}\right)$ contains at least $\frac{2 M L}{L / 1-\delta}=2 M(1-\delta) \geq M$ full frames. The above analysis implies that, once all nodes have completed their epoch $i_{0}$, the neighbor discovery completes with probability at least $1-\epsilon$. Thus we have:

Theorem 13. Let $M=\frac{48 \max \left(2 S, 3 \Delta_{0}\right)}{\rho} \ln \left(\frac{N^{2}}{\epsilon}\right)+1$. Then Algorithm 6 ensures that each node discovers all its neighbors on all channels within $O\left(\left(M+\frac{\Theta}{L}\right) \log \left(M+\frac{\Theta}{L}\right)\left(\frac{L}{1-\delta}\right)\right)$ time-units of $T_{s}$ with probability at least $1-\epsilon$.

Remark (Impact of Assumption 2). Even for very large values of system parameters, namely $N=10^{6}$, $S=10^{6}, \epsilon=10^{-9}$ and $\frac{\Theta}{L}=10^{6}$, Assumption 2 only requires $\delta \leq 2.2 \times 10^{-} 3$ seconds/seconds. This is not very restrictive because, as we mentioned earlier, an ordinary quartz clock has a maximum drift rate of $10^{-6}$ seconds/second.

\section{Summary}

Table 2 summarizes the results of the previous two sections. We now discuss two extensions to our algorithms to enhance their applicability. 
Table 2: A summary of all randomized neighbor discovery algorithms in this work.

\begin{tabular}{|c|c|c|c|c|}
\hline \multicolumn{3}{|c|}{ Assumptions } & \multirow{2}{*}{ Time Complexity* } & \multirow{2}{*}{ Section } \\
\hline Synchrony & Start Times & System Parameters & & \\
\hline Synchronous & Same & $\begin{array}{l}\text { know an estimate for an upper bound } \\
\text { on } \Delta \text {, given by } \Delta_{e s t}\end{array}$ & $O\left(\frac{\max (S, \Delta)}{\rho} \log \left(\Delta_{\text {est }}\right) \log \left(\frac{N}{\epsilon}\right)\right)$ & 3.1 .1 \\
\hline Synchronous & Same & - & $\begin{array}{c}O(M \log M) \text { where } \\
M=\frac{16 \max (S, \Delta)}{\rho} \ln \left(\frac{N^{2}}{\epsilon}\right)\end{array}$ & 3.1 .2 \\
\hline Synchronous & Different & $\begin{array}{l}\text { know an estimate for an upper bound } \\
\text { on } \Delta \text {, given by } \Delta_{e s t}\end{array}$ & $O\left(\frac{\max \left(2 S, \Delta_{\text {est }}\right)}{\rho} \log \left(\frac{N}{\epsilon}\right)\right)$ & 3.2 .1 \\
\hline Synchronous & Different & - & $\begin{array}{c}O((M+\Theta) \log (M+\Theta)) \text { where } \\
M=\frac{16 \max \left(S, \Delta_{0}\right)}{\rho} \ln \left(\frac{N^{2}}{\epsilon}\right)\end{array}$ & 3.2 .2 \\
\hline Asynchronous & Different & $\begin{array}{c}\text { know an estimate for an upper bound } \\
\text { on } \Delta \text {, given by } \Delta_{e s t} \text {, and } \delta \leq \frac{1}{7}\end{array}$ & $O\left(\frac{L \max \left(2 S, 3 \Delta_{e s t}\right)}{\rho} \log \left(\frac{N}{\epsilon}\right)\right)$ & 4.1 \\
\hline Asynchronous & Different & $\delta \leq \frac{1}{48\left\{\log \left(\frac{N S}{\epsilon}\right)+\log \left(1+\frac{\Theta}{L}\right)+5\right\}}$ & $\begin{array}{c}O\left((M L+\Theta) \log \left(M+\frac{\Theta}{L}\right)\right) \text { where } \\
M=\frac{48 \max \left(2 S, 3 \Delta_{0}\right)}{\rho} \ln \left(\frac{N^{2}}{\epsilon}\right)+1\end{array}$ & 4.2 \\
\hline
\end{tabular}

*: In the case of variable start times, the time is measured after the last node has started neighbor discovery

$N$ : total number of CR nodes in the network

$\Delta$ : maximum degree of a node on a channel

$\Delta_{0}$ : smallest power of two greater than or equal to $\Delta$

$S$ : size of the largest available channel set

$\rho$ : minimum span ratio of a link

$\epsilon$ : maximum error probability (user-specified)

$\Theta$ : maximum difference in the start times of two nodes

$L$ : length of a frame

$\delta$ : maximum drift rate of a local clock

\section{Improving Robustness}

In this section, we describe several extensions to our algorithms that increase their robustness in practice.

\subsection{Handling Diverse Propagation Characteristics}

All algorithms described so far have assumed "one-for-all" property that if a link from node $v$ to node $u$ exists on some channel then it also exists on all channels that are present in available channel sets of both $u$ and $v$. That may not hold if different channels have very different propagation characteristics. In case the "one-for-all" property does not hold, we can partition the channels into bands such that the subset of channels within the same band have very similar propagation characteristics. As a result, "one-for-all" holds for each band individually. We say that a link can operate in a band if it can operate on some channel in the band. Each link can then be replaced with multiple links, one for each band in which it can operate. Link for each band then has to be discovered separately. This modification increases the running time of our 
algorithms since more links have to be discovered now. The minimum span-ratio in the network now ranges from $\frac{1}{S}$ to $\frac{W}{S}$ instead of $\frac{1}{S}$ to 1 , where $W$ is the size of the smallest band (in terms of number of channels). Further the term " $\log \left(\frac{N^{2}}{\epsilon}\right)$ " in time complexity expressions is replaced with the term " $\log \left(\frac{N^{2} B}{\epsilon}\right)$ ", where $B$ denote the maximum number of bands in which any link can operate.

\subsection{Handling Lossy Channels}

All algorithms described so far have assumed that channels are reliable in the sense that a node fails to receive a message from its neighbor only due to collision. However, in the real world, a node may fail to receive a message due to other reasons as well such as Gaussian noise or transient interference. Let $\phi$ with $0 \leq \phi<1$ denote the probability that a node fails to receive a message from its neighbor even in the absence of collision. Nodes may not know $\phi$. With lossy channels, the probability that a link is covered by a time-slot (in Algorithm 1, Algorithm 2, Algorithm 3 and Algorithm 4) or an aligned frame-pair (in Algorithm 5 and Algorithm 6) is now multiplied by a factor of $1-\phi$. For example, the probability expression in Lemma 6 now becomes $\frac{\rho(1-\phi)}{8 \max \left(2 S, 3 \Delta_{e s t}\right)}$. It can be verified that this has the effect of increasing the running time of an algorithm by a factor of $\frac{1}{1-\phi}$.

\subsection{Handling Jamming Attacks}

Most of the work on jamming attack $[18,23,38,59]$ is concerned with analyzing or mitigating the impact of jamming attack on communication throughput in the system, and, as such, they assume that neighbor discovery has already completed. Work in [6] presents a suite of neighbor discovery algorithms in the presence of jamming attack. However, unlike our work, the work in [6] assumes a single-hop network and does not provide any theoretical guarantees (algorithms are only evaluated using simulation experiments).

We show that, our neighbor discovery algorithms, with minor modification, are tolerant to jamming attacks by a reactive but "memory-less" jammer under certain assumption. We now describe the jamming model we assume for our analysis.

Jamming model: A jammer operates in rounds. At the beginning of each round, the jammer scans the spectrum and selects a channel to jam during that round from among the channels it observed to be in use in the scan as follows. Let $j$ denote the channel jammed by the jammer in the previous round. Also, let $C$ denote the set of channels the jammer found to be in use in the scan it performed at the beginning of the 
current round. The jammer then makes a uniform random selection of a channel from the set $C \backslash\{j\}$, if non-empty, and jams this channel during the current round.

We assume that slot boundaries and round boundaries need not be aligned even if the system is synchronous. Thus a jamming round can start in the middle of a slot. Note that this works to the advantage of the jammer since (s)he can effectively disrupt transmissions on two channels in a slot by starting a new jamming round near the middle of a slot. It also implies that it is not necessary for the jammer to disrupt the channel $j$ again since it would have already disrupted all transmissions on this channel in the current slot.

We analyze our algorithms under the following assumption:

Assumption 3. The length of a jamming round is at least at large as the length of a slot.

Note that, in the absence of any assumption on the length of a jamming round, a jammer can disrupt all transmissions in a slot, thereby making the jammer too powerful and the neighbor discovery problem impossible to solve. Also, note that, in our jamming model, the neighbor discovery problem can only be solved if there are at least three nodes and at least three channels in the system. Assumption 3 can be relaxed by assuming that a slot can contain up to $k$ jamming rounds, where $k$ is a system parameter. This enables a jammer to disrupt transmissions on up to $2 k$ channels in a slot. Further, a jammer in our model is "memoryless" in the sense that (s)he does not use the results of the prior scans to select a channel to jam. It is possible for a jammer to increases the effectiveness of the jamming attack by using the scan history to select the channel to be disrupted. We leave the analysis of our algorithms under these extensions as a future work.

For ease of exposition and to avoid over-notation, we assume that the network to be homogeneous in the sense that all nodes have the same available channel sets. However, the statements of Theorem 14 and Theorem 15 still hold as long as the available channel set of each node contains at least three channels.

\subsubsection{Synchronous Algorithms}

Recall the events $A(\tau, c), B(\tau, c)$ and $C(\tau, c)$ defined earlier when the analyzing the probability that node $u$ discovers node $v$ on channel $c$ during time-slot $\tau$. We define a new event $D(\tau, c)$ to represent the condition that the transmission from node $v$ to node $u$ on channel $c$ is not jammed during the time-slot $\tau$. For convenience, let $A B C(\tau, c)$ denote the conjunction of the three events $A(\tau, c), B(\tau, c)$ and $C(\tau, c)$. We derive a lower bound on the conditional probability that event $D(\tau, c)$ occurs given that event $A B C(\tau, c)$ occurs. 
Note that the slow-down factor in the running time of a neighbor discovery algorithm that is undergoing a jamming attack is given by the inverse of this conditional probability.

Assume that, at the beginning of the time-slot $\tau$, the jammer was jamming channel $j$. Sometime during $\tau$, the jammer switches to a new channel to jam. We have:

$$
\operatorname{Pr}\{D(\tau, c) \mid A B C(\tau, c)\}=\left(\frac{S-1}{S}\right) \cdot \operatorname{Pr}\{E(\tau, c) \mid A B C(\tau, c)\} \cdot\left(\frac{1}{2}\right)
$$

where $E(\tau, c)$ denotes the event that some node in the system transmits on a channel other than $c$ and $j$ during the time-slot $\tau$ with $c \neq j$. In the above expression, the first term (given by $\frac{S-1}{S}$ ) captures the probability that $c \neq j$ and the third term (given by $\frac{1}{2}$ ) the probability that the jammer chooses a channel other than $c$ to jam.

We next compute the conditional probability that the event $E(\tau, c)$ occurs given that the event $A B C(\tau, c)$ occurs. We partition the set of nodes in the system into three mutually-exclusive groups: $G_{1}, G_{2}$ and $G_{3}$. Group $G_{1}$ contains nodes $u$ and $v$, group $G_{2}$ contains nodes that are neighbors of node $u$ except for node $v$, and group $G_{3}$ contains the remaining nodes.

Let $\mathcal{A}$ denote the set of all available channels. (Note that, by our assumption, all nodes have identical available channel sets.) Also, let $F(\tau, c, w)$ denote the event that node $w$ does not transmit on a channel in $\mathcal{A} \backslash\{c, j\}$ during time-slot $\tau$. Since nodes act independently of each other, we have:

$$
\begin{aligned}
\operatorname{Pr}\{E(\tau, c) \mid A B C(\tau, c)\}= & 1-\prod_{i \in\{1,2,3\}} \prod_{w \in G_{i}} \operatorname{Pr}\{F(\tau, c, w) \mid A B C(\tau, c)\} \\
& \left\{\forall w: w \in G_{1}: \operatorname{Pr}\{F(\tau, c, w) \mid A B C(\tau, c)\}=1\right\} \\
= & 1-\prod_{i \in\{2,3\}} \prod_{w \in G_{i}} \operatorname{Pr}\{F(\tau, c, w) \mid A B C(\tau, c)\} \\
& \left\{\begin{array}{l}
\forall w: w \in G_{3}: \operatorname{Pr}\{F(\tau, c, w) \mid A B C(\tau, c)\}=\operatorname{Pr}\{F(\tau, c, w)\} \\
\because \operatorname{nodes} \operatorname{act} \text { independently }
\end{array}\right\} \\
= & 1-\left(\prod_{w \in G_{2}} \operatorname{Pr}\{F(\tau, c, w) \mid A B C(\tau, c)\}\right) \cdot\left(\prod_{w \in G_{3}} \operatorname{Pr}\{F(\tau, c, w)\}\right)
\end{aligned}
$$

Let $p$ denote the placeholder for transmission probability. Note that $p=\min \left(\frac{1}{2}, \frac{S}{\Delta_{e s t}}\right)$. For our 
analysis, we focus on time-slots for which $\Delta_{e s t}$ satisfies the following condition:

$$
\Delta_{e s t} \leq 2(N-2)
$$

We show that the above-condition trivially holds for three out of four synchronous algorithms, namely Algorithm 1, Algorithm 2 and Algorithm 4. For Algorithm 3, in which $\Delta_{e s t}$ is an "external" parameter, we state it as an explicit assumption. In the three synchronous algorithms (in which nodes estimate degree), for the time-slots used to show that neighbor discovery completes with arbitrarily high probability, note that $\Delta_{e s t}$ is the smallest power of two that is greater than or equal to either $\Delta(u, c)$ or $\Delta$. In either case, we have:

$$
\begin{aligned}
\frac{\Delta_{e s t}}{2}<\Delta \Longrightarrow & \frac{\Delta_{\text {est }}}{2} \leq \Delta-1 \\
& \{\Delta \leq N-1\} \\
\Longrightarrow & \frac{\Delta_{\text {est }}}{2} \leq(N-1)-1=N-2
\end{aligned}
$$

We now derive the conditional probability $\operatorname{Pr}\{F(\tau, c, w) \mid A B C(\tau, c)\}$. There are two cases depending on whether $w \in G_{2}$ or $w \in G_{3}$. The derivation of the following result is relatively straightforward has been moved to the appendix (see Section D).

$$
\operatorname{Pr}\{F(\tau, c, w) \mid A B C(\tau, c)\}= \begin{cases}1-\frac{S-2}{\frac{S}{p}-1} & : w \in G_{2} \\ 1-\frac{S-2}{\frac{S}{p}} & : w \in G_{3}\end{cases}
$$

Using (19) and (21), we have:

$$
\begin{aligned}
\operatorname{Pr}\{E(\tau, c) \mid A B C(\tau, c)\} & =1-\prod_{w \in G_{2}}\left(1-\frac{S-2}{\frac{S}{p}-1}\right) \cdot \prod_{w \in G_{3}}\left(1-\frac{S-2}{\frac{S}{p}}\right) \\
& \geq 1-\prod_{w \in G_{2}}\left(1-\frac{S-2}{\frac{S}{p}}\right) \cdot \prod_{w \in G_{3}}\left(1-\frac{S-2}{\frac{S}{p}}\right) \\
& =1-\prod_{w \in G_{2} \cup G_{3}}\left(1-\frac{S-2}{\frac{S}{p}}\right)=1-\left(1-p \cdot \frac{S-2}{S}\right)^{N-2}
\end{aligned}
$$



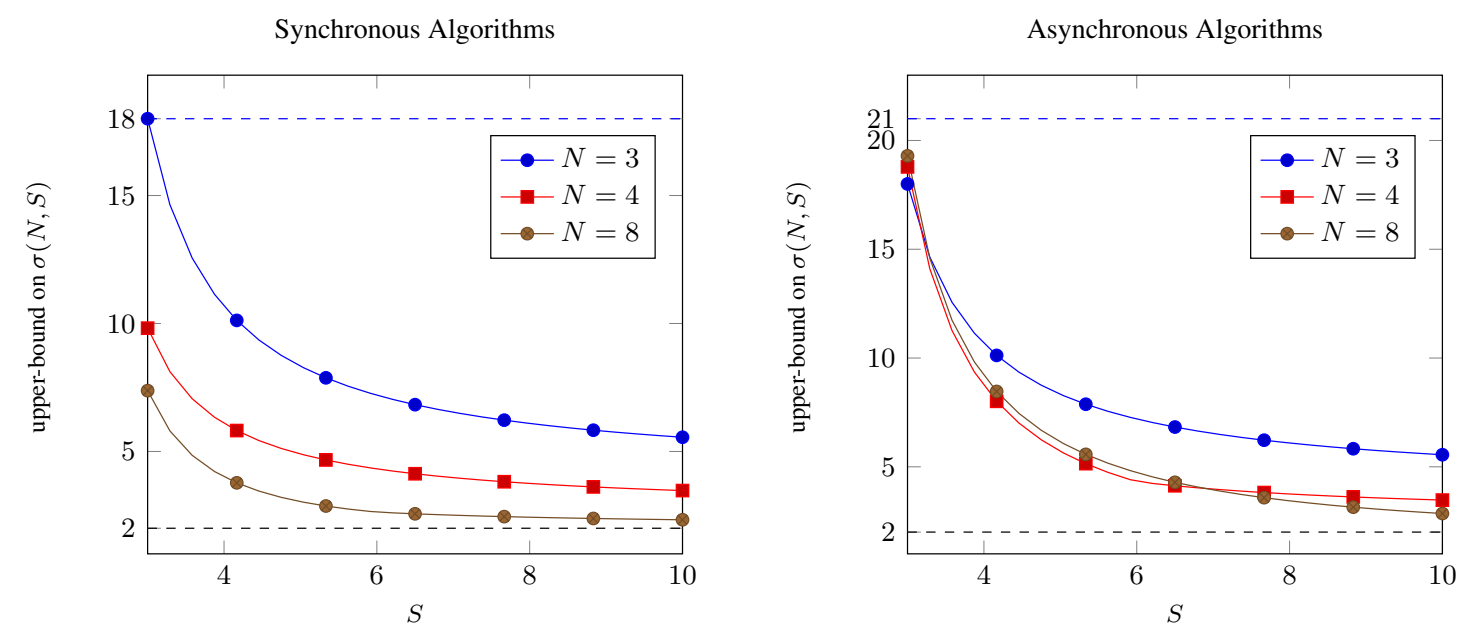

Figure 3: Upper bound on slow-down factor as a function of $N$ and $S$.

Analyzing the two cases corresponding to when $p=\frac{1}{2}$ and when $p=\frac{S}{\Delta_{e s t}}$ separately, we can show that:

$$
\operatorname{Pr}\{D(\tau, c) \mid A B C(\tau, c)\} \geq \frac{1}{18}
$$

The proof has been moved to the appendix (see Section D). Let $\sigma(N, S)$ denote the slow-down factor experienced by a neighbor discovery algorithm that is undergoing a jamming attack. The above analysis implies that $\sigma(N, S) \leq 18$ for all $N \geq 3$ and $S \geq 3$. For sufficiently large values of $N$ and $S$, we show that the slow-down factor is much smaller. Specifically, we show that:

$$
\lim _{\substack{N \rightarrow \infty \\ S \rightarrow \infty}} \sigma(N, S) \leq 2
$$

Again, the proof involves analyzing the cases corresponding to when $p=\frac{1}{2}$ and when $p=\frac{S}{\Delta_{e s t}}$ separately and has been moved to the appendix (see Section D). Finally, we have:

Theorem 14. The running time of the synchronous algorithms increases by a factor of 18 in the worst case. Further, when $N$ and $S$ are sufficient large, the running time only increases by a factor of at most 2 .

Note that the slow-down factor of 18 represents the worst-case and only occurs for a "small" system which intuitively is easier to jam. As $N$ and $S$, increases, the slow-down factor decreases rapidly as depicted in Fig. 3. 


\subsubsection{Asynchronous Algorithms}

In the asynchronous case, we require a minor change to Algorithm 5 and Algorithm 6. Namely, when a node is in transmit mode, it makes a uniform selection of channel at the beginning of each slot, rather than at the beginning of each frame. We also assume that the length of a jamming round is at least the maximum length of a slot (measured in real-time), which is given by $\frac{L}{3(1-\delta)}$.

Consider the link from node $v$ to node $u$. Consider a legal pair of frames $\langle f, g\rangle$ with node $(f)=v$ and $\operatorname{node}(g)=u$. Let $\operatorname{slot}(f, g)$ denote the slot of $f$ that lies completely within $g$. (If there are multiple such slots, then choose one arbitrarily.) For analyzing the jamming attack, we narrow the period during which no transmission by other neighbors of $u$ interferes with $v$ 's transmission during $\operatorname{slot}(f, g)$. This, in conjunction with the assumption made on the minimum length of jamming round, ensures that the set of channels a jammer observes to be in use in two different scans do not have any correlation. Intuitively, this is because two consecutive scans by a jammer will involve two different slots of any node and, in transmit mode, a node now chooses the channel on which to transmit at the beginning of a slot (rather than a frame).

Consider events $\hat{A}(f, g, c), \hat{B}(f, g, c)$ and $\hat{C}(f, g, c)$. To analyze our algorithms under jamming attack, we replace the event $\hat{A}(f, g, c)$ with a new event $\hat{A}^{\prime}(f, g, c)$ which denotes the condition that $u$ transmits on $c$ during $\operatorname{slot}(f, g)$. Further, we replace the event $\hat{C}(f, g, c)$ with a new event $\hat{C}^{\prime}(f, g, c)$ which denotes the condition that no neighbor of $u$ besides $v$ transmits on $c$ during any of its slots that overlaps with $\operatorname{slot}(f, g)$. Clearly, in the absence of jamming attack, $u$ will discover $v$ during frame $g$ if events $\hat{A}^{\prime}(f, g, c), \hat{B}(f, g, c)$ and $\hat{C}^{\prime}(f, g, c)$ occur. Analogous to Lemma 5, it can be easily shown that a single slot of a node can overlap with at most three slots of another node. Note that these three slots can come from at most two different frames. It can be verified that (11) holds for $\hat{A}^{\prime}(f, g, c)$ and (13) holds for $\hat{C}^{\prime}(f, g, c)$ (for the latter, see Section E in the appendix).

Analogous to events $A B C(\tau, c), D(\tau, c)$ and $E(\tau, c)$ and $F(\tau, c, w)$ define in the synchronous case, we can define events $A \hat{B} C(f, g, c), \hat{D}(f, g, c), \hat{E}(f, g, c)$ and $\hat{F}(f, g, c$,$) , respectively. Most of the analysis for$ the synchronous case is also applicable to the asynchronous case. Specifically, when $p=\frac{1}{2}$, the analysis and results for the synchronous case also apply to the asynchronous case. However, when $p=\frac{S}{3 \Delta_{e s t}}$, the results for the asynchronous case are slightly different from that for the synchronous case. Since the analysis is very similar to that for the synchronous case, it has been moved to the appendix (see Section E). In particular, we can show the following: (i) for all $N S$, where $N \geq 3$ and $S \geq 3, \sigma(N, S) \leq 21$ and 
(ii) $\lim _{\substack{N \rightarrow \infty \\ S \rightarrow \infty}} \sigma(N, S) \leq 2$. Finally, we have the following result:

Theorem 15. The running time of the asynchronous algorithms increases by a factor of 21 in the worst case. Further, when $N$ and $S$ are sufficient large, the running time only increases by a factor of at most 2.

Again, as in the synchronous case, the slow-down factor of 21 represents the worst-case and only occurs for a "small" system which intuitively is easier to jam. As $N$ and $S$, increases, the slow-down factor decreases rapidly as depicted in Fig. 3.

\section{Related Work}

A large number of neighbor discovery algorithms have been proposed in the literature. To our knowledge, most of the algorithms suffer from one or more of the following limitations:

- Single-Channel Network: Several neighbor discovery algorithms have been proposed for a (traditional) single channel wireless network (e.g., $[3,4,9,24,25,31,42,51,56-58,61,64,67,69])$. Some of these neighbor discovery algorithms, such as those proposed in $[56,58]$, can be extended to work for a multi-channel network (including a heterogeneous network). The main idea is as follows. Let the collective set of all channels over which radio nodes in the network are capable of operating be referred to as the universal channel set. The main idea is to execute a separate instance of singlechannel neighbor discovery algorithm on all channels in the universal channel set concurrently by interleaving their executions. A node only participates in instances that are associated with channels in its available channel set. However, this simple approach has several disadvantages. First, it requires that all nodes have to agree on the composition of the universal channel set. Second, the time complexity of the algorithm for multi-channel network (obtained as above) will always be linear in the size of the universal channel set. This is true even if the available channel set of all nodes contain a single common channel (an extreme case). In many cases, the available channel sets of nodes may be much smaller than the universal channel set. Third, all nodes should start executing the algorithm at the same time. Otherwise, different nodes may tune to different channels in the same time slot, thereby causing the multi-channel neighbor discovery algorithm to fail. As such, this transformation does not work for an asynchronous system. In comparison, in our algorithms, nodes do not need to 
agree on a universal channel set and the time complexity of our algorithms depends on the "degree of heterogeneity" in the network.

- Single-Hop Network: Neighbor discovery algorithms in $[2,5,6]$ consider a single-hop network in which all nodes can communicate with each other directly. Our algorithms, on the other hand, work even for a multi-hop network.

- Synchronous System: Neighbor discovery algorithms in $[17,33,55]$ assume a time-slotted synchronous system. Work in $[35,44,66]$ assume a synchronous system in which all nodes initiate neighbor discovery at the same time. Moreover, the proposed algorithms are deterministic in nature, and have high time complexity that depends on the product of network size and universal channel set size. In contrast, our focus in this work is on developing algorithms for an asynchronous system that have lower running time (albeit provide only probabilistic guarantees).

- Homogeneous Channel Availability: Neighbor discovery algorithms in [2, 17, 34] assume homogeneous channel availability (all channels are available to all nodes). Our algorithms, on the other hand, work even when different nodes have different subsets of channels available for communication, and, further, their running time adapts to the "degree of heterogeneity" in the network.

- Little or No Theoretical Guarantees: Work in $[48,60]$ only evaluate the proposed neighbor discovery algorithms using experiments; no attempt is made to provide any theoretical (deterministic or probabilistic) guarantees. Work in [6] provides a suite of neighbor discovery algorithms that can tolerate jamming attacks. However, it does not provide any theoretical guarantees either (algorithms are evaluated using only simulation experiments). In our work, in contrast, we analyze all our algorithms theoretically and establish probabilistic bounds on their performance.

Besides the related work mentioned above, Raniwala and Chiueh propose a neighbor discovery algorithm for a multi-channel wireless network in [49] but their work assumes that each node has multiple interfaces. Law et al. describe a neighbor discovery algorithm for constructing a scatternet in [36]. Salondis et al. describe an algorithm for two-node link formation in [52], which is then used as a building block for forming a scatternet in [53]. A scatternet is an ad hoc network consisting of two or more piconets; a piconet is an ad hoc network consisting two or more (up to eight) Bluetooth-enabled devices with one device acting 
as master and remaining devices acting as slaves. Work in $[8,17,19,20,28,30,41,43]$ addresses the closelyrelated problem of rendezvous between two CR nodes. Intuitively, the rendezvous problem between two nodes involves ensuring that the two nodes tune to the same channel at the same time, which is necessary for nodes to discover each other. However, as opposed to the problem considered in this work, the rendezvous problem does not consider the effect of possible simultaneous transmissions by other neighboring nodes at the same, which may cause collisions thereby preventing a link from being discovered.

\section{Conclusion}

In this work, we have proposed several randomized neighbor discovery algorithms for an $\mathrm{M}^{2} \mathrm{HeW}$ network both for synchronous and asynchronous systems under a variety of assumptions. Our algorithms guarantee success with arbitrarily high probability. All our algorithms are designed to minimize the amount of knowledge that nodes need to possess a priori before they start executing the neighbor discovery algorithm.

As a future work, we plan to develop neighbor discovery algorithms for an $\mathrm{M}^{2} \mathrm{HeW}$ network that are robust to even more powerful jammer who can either disrupt multiple channels in a round or can use the scan history to select one or more channels to disrupt. We also plan to develop robust randomized algorithms for solving other communication problems for an $\mathrm{M}^{2} \mathrm{HeW}$ network such as broadcasting and convergecasting that guarantee success with arbitrarily high probability.

\section{Acknowledgments}

The authors would like to thank anonymous reviewers for comments and suggestions that helped in improving the manuscript.

\section{References}

[1] I. F. Akyildiz, B. F. Lo, and R. Balakrishnan. Cooperative Spectrum Sensing in Cognitive Radio Networks: A Survey. Physical Communication, 4(1):40-62, March 2011.

[2] G. Alonso, E. Kranakis, C. Sawchuk, R. Wattenhofer, and P. Widmayer. Probabilistic Protocols for Node Discovery in Ad-Hoc Multi-Channel Broadcast Networks. In Proceedings of the 2nd Annual Conference on Adhoc Networks and Wireless (ADHOCNOW), pages 101-115, 2003. 
[3] G. Alonso, E. Kranakis, R. Wattenhofer, and P. Widmayer. Probabilistic Protocols for Node Discovery in Ad-Hoc, Single Broadcast Channel Networks. In Proceedings of the International Parallel and Distributed Processing Symposium (IPDPS), Nice, France, April 2003.

[4] X. An and R. Hekmat. Self-Adaptive Neighbor Discovery in Ad Hoc Networks with Directional Antennas. In Proceedings of the 16th IST Summit on Mobile and Wireless Communications, pages $1-5,2007$.

[5] C. Arachchige, S. Venkatesan, and N. Mittal. An Asynchronous Neighbor Discovery Algorithm for Cognitive Radio Networks. In Proceedings of the 3rd IEEE Symposia on New Frontiers in Dynamic Spectrum Access Networks (DySPAN), Chicago, Illinois, USA, 2008.

[6] A. Asterjadhi and M. Zorzi. JENNA: A Jamming Evasive Network-coding Neighbor-discovery Algorithm for Cognitive Radio Networks. In Proceedings of the 2010 IEEE International Conference on Communications Workshops, Capetown, South Africa, May 2010.

[7] L. Bao and J. J. Garcia-Luna-Aceves. Transmission Scheduling in Ad Hoc Networks with Directional Antennas. In Proceedings of the 8th Annual ACM/IEEE International Conference on Mobile Computing and Networking (MobiCom), pages 48-58, Atlanta, Georgia, USA, September 2002.

[8] K. Bian and J.-M. Park. Maximizing Rendezvous Diversity in Rendezvous Protocols for Decentralized Cognitive Radio Networks. IEEE Transactions on Mobile Computing (TMC), 12(7):1294-1307, July 2013 .

[9] S. A. Borbash, A. Ephremides, and M. J. McGlynn. An Asynchronous Neighbor Discovery Algorithm for Wireless Sensor Networks. Ad Hoc Networks, 5(7):998-1016, September 2007.

[10] A. Boukerche. Handbook of Algorithms For Wireless Networking and Mobile Computing. CRC Press, 2005.

[11] A. Boukerche. Algorithms and Protocols for Wireless, Mobile Ad Hoc Networks, volume 77. John Wiley and Sons, Incorporated, 2008.

[12] A. Boukerche, C. Rezende, and R. W. Pazzi. Improving Neighbor Localization in Vehicular Ad Hoc Networks to Avoid Overhead from Periodic Messages. In Proceedings of the IEEE Global Telecommunications Conference (GLOBECOM), November 2009. 
[13] R. W. Broderson, A. Wolisz, D. Cabric, S. M. Mishra, and D. Willkomm. CORVUS: A Cognitive Radio Approach for Usage of Virtual Unlicensed Spectrum. Available at http://bwrc.eecs . berkeley•edu/Research/MCMA/CR \_White\_paper\_final1.pdf.

[14] G. Cafaro, N. Correal, and D. Taubenheim. A $100 \mathrm{MHz}-2.5 \mathrm{GHz}$ CMOS Transceiver in an Experimental Cognitive Radio System. In Proceedings of the SDR (Software Defined Radio) Technical Conference and Product Exposition, Denver, Colorado, USA, November 2007.

[15] M. Cesana, F. Cuomo, and E. Ekici. Routing in Cognitive Radio Networks: Challenges and Solutions. Ad Hoc Networks, 9(3):228-248, May 2010.

[16] Y.-L. Chang and C.-C. Hsu. Routing in Wireless/Mobile Ad-Hoc Networks via Dynamic Group Construction. Mobile Networks and Applications (MONET), 5(1):27-37, March 2000.

[17] C.-M. Chao and C.-Y. Hsu. Supporting Fast Neighbor Discovery for Cognitive Radio Networks. In Proceedings of the International Conference on Computational Problem-Solving (ICCP), pages 123126, October 2013.

[18] C. Chen, M. Song, C.S. Xin, and J. Backens. A Game-Theoretical Anti-Jamming Scheme for Cognitive Radio Networks. IEEE Network, 27(3):22-27, May/June 2013.

[19] L. Chen, K. Bian, L. Chen, C. Liu, J.-M. J. Park, and X. Li. A Group-Theoretic Framework for Rendezvous in Heterogeneous Cognitive Radio Networks. In Proceedings of the 15th ACM International Symposium on Mobile Ad Hoc Networking and Computing (MobiHoc), pages 165-174, August 2014.

[20] L. Chen, K. Bian, and M. Zheng. Heterogeneous Multi-Channel Neighbor Discovery for Mobile Sensing Applications: Theoretical Foundation and Protocol Design. In Proceedings of the 15th ACM International Symposium on Mobile Ad Hoc Networking and Computing (MobiHoc), pages 307-316, August 2014.

[21] Y. Choi, M. Khan, V. S. Anil Kumar, and G. Pandurangan. Energy-Optimal Distributed Algorithms for Minimum Spanning Trees. IEEE Journal on Selected Areas in Communications (JSAC), 27(7):12971304, September 2009.

[22] R. Choudhury, X. Yang, R. Ramanathan, and N. Vaidya. Using Directional Antennas for Medium Access Control in Ad Hoc Networks. In Proceedings of the 8th Annual ACM/IEEE International 
Conference on Mobile Computing and Networking (MobiCom), pages 59-70, Atlanta, Georgia, USA, September 2002.

[23] K. Dabcevic, A. Betancourt, L. Marcenaro, and C. S. Regazzoni. A Fictitious Play-Based GameTheoretical Approach to Alleviating Jamming Attacks for Cognitive Radios. In Proceedings of the IEEE International Conference on Acoustics, Speech and Signal Processing (ICASSP), pages 81588162, Florence, Italy, May 2014.

[24] P. Dutta and D. Culler. Practical Asynchronous Neighbor Discovery and Rendezvous for Mobile Sensing Applications. In Proceedings of the ACM Conference on Embedded Networked Sensor Systems (SenSys), pages 71-84, November 2008.

[25] V. Dyo and C. Mascolo. Efficient Node Discovery in Mobile Wireless Sensor Networks. In Proceedings of the IEEE international Conference on Distributed Computing in Sensor Systems (DCOSS), pages 478-485, Santorini Island, Greece, June 2008.

[26] S. Gandham, M. Dawande, and R. Prakash. Link Scheduling in Wireless Sensor Networks: Distributed Edge-Coloring Revisited. Journal of Parallel and Distributed Computing (JPDC), 68(8):1122-1134, August 2008.

[27] S. Gandham, Y. Zhang, and Q. Huang. Distributed Time-Optimal Scheduling for Convergecast in Wireless Sensor Networks. Computer Networks (COMNET), 52:610-629, 2008.

[28] R. Gandhi, C.-C. Wang, and Y. C. Hu. Fast Rendezvous for Multiple Clients for Cognitive Radios using Coordinated Channel Hopping. In Proceedings of the 8th IEEE Communications Society Conference on Sensor, Mesh and Ad Hoc Communications and Networks (SECON), pages 434-442, June 2012.

[29] A. Gonga, T. Charalambous, and M. Johansson. Neighbor Discovery in Multichannel Wireless Clique Networks: An Epidemic Approach. In Proceedings of the 10th IEEE International Conference on Mobile Ad Hoc and Sensor Systems (MASS), pages 131-135, 2013.

[30] Z. Gu, Q.-S. Hua, Y. Wang, and F. C. M. Lau. Nearly Optimal Asynchronous Blind Rendezvous Algorithm for Cognitive Radio Networks. In Proceedings of the 10th IEEE Communications Society Conference on Sensor, Mesh and Ad Hoc Communications and Networks (SECON), pages 371-379, June 2013. 
[31] E. B. Hamida, G. Chelius, A. Busson, and E. Fleury. Neighbor Discovery in Multi-Hop Wireless Networks: Evaluation and Dimensioning with Interferences Considerations. Discrete Mathematics and Theoretical Computer Science, 10(2):87-114, 2008.

[32] W. Heinzelman, A. Chandrasekaran, and H. Balakrishnan. Energy Efficient Communication Protocol for Wireless Microsensor Networks. In Proceedings of the Hawaii International Conference on Systems Sciences, pages 3005-3014, Maui, Hawaii, USA, January 2000.

[33] V. Iyer, A. Pruteanu, and S. Dulman. NetDetect: Neighborhood Discovery in Wireless Networks Using Adaptive Beacons. In Proceedings of the 5th IEEE International Conference on Self-Adaptive and Self-Organizing Systems (SASO), pages 31-40, October 2011.

[34] N. Karowski, A. C. Viana, and A. Wolisz. Optimized Asynchronous Multi-Channel Neighbor Discovery. In Proceedings of the IEEE Conference on Computer Communications (INFOCOM), pages 536-540, 2011.

[35] S. Krishnamurthy, M. Thoppian, S. Kuppa, R. Chandrasekaran, N. Mittal, S. Venkatesan, and R. Prakash. Time-efficient Distributed Layer-2 Auto-configuration for Cognitive Radio Networks. Computer Networks (COMNET), 52(4):831-849, March 2008.

[36] C. Law, A. K. Mehta, and K.-Y. Siu. Performance of a new Bluetooth Scatternet Formation Protocol. In Proceedings of the 2nd ACM International Symposium on Mobile Ad Hoc Networking and Computing (MobiHoc), pages 183-192, 2001.

[37] L. Li, J. Y. Halpern, P. Bahl, Y.-M. Wang, and R. Wattenhofer. A Cone-Based Distributed TopologyControl Algorithm for Wireless Multi-Hop Networks. IEEE/ACM Transactions on Networking, 13(1):147-159, 2005.

[38] X. Li and W. Cadeau. Anti-Jamming Performance of Cognitive Radio Networks. In Proceedings of the 45th Annual Conference on Information Systems and Sciences (CISS), Baltimore, Maryland, USA, March 2011.

[39] H. Lim and C. Kim. Multicast Tree Construction and Flooding in Wireless Ad Hoc Networks. In Proceedings of the 3rd ACM International Workshop on Modeling, Analysis and Simulation of Wireless and Mobile Systems (MSWiM), pages 61-68, Boston, Massachusetts, USA, August 2000. 
[40] C. R. Lin and M. Gerla. Adaptive Clustering for Mobile Wireless Networks. IEEE Journal on Selected Areas in Communications (JSAC), 15(7):1265-1275, September 1997.

[41] H. Liu, Z. Lin, X. Chu, and Y.-W. Leung. Jump-Stay Rendezvous Algorithm for Cognitive Radio Networks. IEEE Transactions on Parallel and Distributed Systems (TPDS), 23(10):1867-1881, October 2012.

[42] M. J. McGlynn and S. A. Borbash. Birthday Protocols for Low Energy Deployment and Flexible Neighbor Discovery in Ad Hoc Wireless Networks. In Proceedings of the 2nd ACM International Symposium on Mobile Ad Hoc Networking and Computing (MobiHoc), pages 137-145, Long Beach, California, USA, 2001.

[43] J. Misic, V. B. Misic, M. S. I. Khan, and M. M. Rahman. Properties of Blind Rendezvous in Channel Hopping Cognitive Piconets. In Proceedings of the IEEE 78th Vehicular Technology Conference (VTC Fall), September 2013.

[44] N. Mittal, S. Krishnamurthy, R. Chandrasekaran, S. Venkatesan, and Y. Zeng. On Neighbor Discovery in Multi-Channel Cognitive Radio Networks. Journal of Parallel and Distributed Computing (JPDC), 69(7):623-637, July 2009.

[45] N. Mittal, Y. Zeng, S. Venkatesan, and R. Chandrasekaran. Randomized Distributed Algorithms for Neighbor Discovery in Multi-hop Multi-channel Heterogeneous Wireless Networks. In Proceedings of the 31st IEEE International Conference on Distributed Computing Systems (ICDCS), pages 57-66, Minneapolis, Minnesota, USA, June 2011.

[46] K. Nakano and S. Olariu. Randomized Leader Election Protocols in Radio Networks with no Collision Detection. In Proceedings of the Annual International Symposium on Algorithms and Computation (ISAAC), pages 362-373, Taipei, Taiwan, December 2000.

[47] R. Prakash. Unidirectional Links Prove Costly in Wireless Ad Hoc Networks. In Proceedings of the 3rd International Workshop on Discrete Algorithms and Methods for Mobile Computing and Communications (DIAL-M), pages 15-22, Seattle, Washington, USA, August 1999. 
[48] L. Raju, S. Ganu, B. Anepu, I. Seskar, and D. Raychaudhuri. Beacon Assisted Discovery Protocol (BEAD) for Self-Organizing Hierarchical Ad-Hoc Networks. In Proceedings of the IEEE Global Telecommunications Conference (GLOBECOM), volume 3, pages 1676-1680, 2004.

[49] A. Raniwala and T. c. Chiueh. Architecture and Algorithms for an IEEE 802.11-based Multi-Channel Wireless Mesh Network. In Proceedings of the 24th IEEE Conference on Computer Communications (INFOCOM), pages 2223-2234, Miami, Florida, USA, March 2005.

[50] M. H. Rehmania, A. C. Vianab, H. Khalifec, and S. Fdidaa. SURF: A Distributed Channel Selection Strategy for Data Dissemination in Multi-Hop Cognitive Radio Networks. Computer Communications (COMCOM), 36(10-11):1172-1185, June 2013.

[51] A. Russell, S. Vasudevan, B. Wang, W. Zeng, X. Chen, and W. Wei. Neighbor Discovery in Wireless Networks with Multipacket Reception. IEEE Transactions on Parallel and Distributed Systems (TPDS), 2014. PrePrint, doi:10.1109/TPDS.2014.2321157.

[52] T. Salonidis, P. Bhagwat, and L. Tassiulas. Proximity Awareness and Fast Connection Establishment in Bluetooth. In Proceedings of the 1st ACM International Symposium on Mobile Ad Hoc Networking and Computing (MobiHoc), pages 141-142, 2000.

[53] T. Salonidis, P. Bhagwat, L. Tassiulas, and R. LaMaire. Distributed Topology Construction of Bluetooth Personal Area Networks. In Proceedings of the IEEE Conference on Computer Communications (INFOCOM), pages 1577-1586, April 2001.

[54] Y. Song and J. Xie. A Distributed Broadcast Protocol in Multi-Hop Cognitive Radio Ad Hoc Networks without a Common Control Channel. In Proceedings of the IEEE Conference on Computer Communications (INFOCOM), pages 2273-2281, March 2012.

[55] G. Sun, F. Wu, X. Gao, and G. Chen. PHED: Pre-Handshaking Neighbor Discovery Protocols in full duplex wireless ad hoc networks. In Proceedings of the IEEE Global Telecommunications Conference (GLOBECOM), pages 584-590, Dec 2012.

[56] S. Vasudevan, M. Adler, D. Goeckel, and D. Towsley. Efficient Algorithms for Neighbor Discovery in Wireless Networks. IEEE/ACM Transactions on Networking, 21(1):69-83, February 2013. 
[57] S. Vasudevan, J. Kurose, and D. Towsley. On Neighbor Discovery in Wireless Networks with Directional Antennas. In Proceedings of the 24th IEEE Conference on Computer Communications (INFOCOM), pages 2502-2512, Miami, Florida, USA, March 2005.

[58] S. Vasudevan, D. Towsley, D. Goeckel, and R. Khalili. Neighbor Discovery in Wireless Networks and the Coupon Collector's Problem. In Proceedings of the 15th Annual ACM/IEEE International Conference on Mobile Computing and Networking (MobiCom), pages 181-192, Beijing, China, 2009.

[59] B. Wang, Y. Wu, K. J. R. Liu, and T. C Clancy. An Anti-Jamming Stochastic Game for Cognitive Radio Networks. IEEE Journal on Selected Areas in Communications (JSAC), 29(4):877-889, April 2011.

[60] F. Wang, S. Sun, L. Li, W. Guo, and Y. Ma. Neighbor Discovery based on Group Frequency Hopping without Common Control Channel for Cognitive Radio Ad Hoc Networks. In Proceedings of the 15th IEEE International Conference on Communication Technology (ICCT), pages 483-487, Guilin, China, 2013.

[61] K. Wang, X. Mao, and Y. Liu. BlindDate: A Neighbor Discovery Protocol. IEEE Transactions on Parallel and Distributed Systems (TPDS), 26(4):949-959, April 2015.

[62] R. Wattenhofer, L. Li, P. Bahl, and Y.-M. Wang. Distributed Topology Control for Power Efficient Operation in Multihop Wireless Ad Hoc Networks. In Proceedings of the 20th IEEE Conference on Computer Communications (INFOCOM), pages 1388-1397, Anchorage, Alaska, USA, April 2001.

[63] A. Willig, N. Karowski, and J.-H. Hauer. Passive Discovery of IEEE 802.15.4-based Body Sensor Networks. Annual Conference on Adhoc Networks and Wireless (ADHOCNOW), 8(7):742-754, September 2010 .

[64] D. Yang, J. Shin, J. Kim, and C. Kim. Asynchronous Probing Scheme for the Optimal Energy-Efficient Neighbor Discovery in Opportunistic Networking. In Proceedings of the IEEE International Conference on Pervasive Computing and Communications (PerCom), pages 1-4, Galveston, Texas, USA, March 2009.

[65] T. Yücek and H. Arslan. A Survey of Spectrum Sensing Algorithms for Cognitive Radio Applications. IEEE Communications Surveys \& Tutorials, 11(1):116-130, 2009. 
[66] Y. Zeng, N. Mittal, S. Venkatesan, and R. Chandrasekaran. Fast Neighbor Discovery with Lightweight Termination Detection in Heterogeneous Cognitive Radio Networks. In Proceedings of the 9th International Symposium on Parallel and Distributed Computing (ISPDC), pages 149-156, Istanbul, Turkey, July 2010.

[67] L. Zhang, J. Luo, and D. Guo. Neighbor Discovery for Wireless Networks via Compressed Sensing. Performance Evaluation, 70(7):457-471, 2013.

[68] R. Zhang and Y. Zhang. Wormhole-Resilient Secure Neighbor Discovery in Underwater Acoustic Networks. In Proceedings of the 29th IEEE Conference on Computer Communications (INFOCOM), pages 1-9, March 2010.

[69] R. Zheng, J. Hou, and L. Sha. Asynchronous Wakeup for Ad Hoc Networks. In Proceedings of the 4th ACM International Symposium on Mobile Ad Hoc Networking and Computing (MobiHoc), pages 35-45, Annapolis, Maryland, USA, June 2003.

\section{A Omitted Proofs from Section 3.1.1}

\section{A.1 Computing the probability of occurrence of $A(\tau, c)$}

We have:

$$
\begin{aligned}
\operatorname{Pr}\{A(\tau, c)\} & =(v \text { selects } c \text { at the beginning of } \tau) \wedge(v \text { chooses to transmit during } \tau) \\
& =\frac{1}{|\mathcal{A}(v)|} \times \min \left(\frac{1}{2}, \frac{|\mathcal{A}(v)|}{2^{k}}\right) \\
& =\min \left(\frac{1}{2|\mathcal{A}(v)|}, \frac{1}{2^{k}}\right) \\
& \left\{\text { using }(2), 2^{k-1} \leq \Delta(u, c) \text { which implies } 2^{k} \leq 2 \Delta(u, c)\right\} \\
& \geq \frac{1}{\max \{2|\mathcal{A}(v)|, 2 \Delta(u, c)\}} \\
& \geq \frac{1}{2 \max (S, \Delta)}
\end{aligned}
$$




\section{A.2 Computing the probability of occurrence of $B(\tau, c)$}

We have:

$$
\begin{aligned}
\operatorname{Pr}\{B(\tau, c)\} & =(u \text { selects } c \text { at the beginning of } \tau) \wedge(u \text { chooses to listen during } \tau) \\
& =\frac{1}{|\mathcal{A}(u)|} \times\left\{1-\min \left(\frac{1}{2}, \frac{|\mathcal{A}(u)|}{2^{k}}\right)\right\} \\
& \{\min (x, y) \leq x\} \\
& \geq \frac{1}{|\mathcal{A}(u)|} \times\left(1-\frac{1}{2}\right)=\frac{1}{2|\mathcal{A}(u)|}
\end{aligned}
$$

\section{A.3 Computing the probability of occurrence of $C(\tau, c)$}

Let $\mathcal{N}(u, c)$ denote the set of neighbors of $u$ on $c$. Note that, if $\mathcal{N}(u, c)$ only contains $v$, then $\operatorname{Pr}\{C(\tau, c)\}=$ 1. Otherwise, we have:

$$
\begin{aligned}
& \operatorname{Pr}\{C(\tau, c)\}=\prod_{\substack{w \in \mathcal{N}(u, c) \\
w \neq v}} \operatorname{Pr}(w \text { does not transmit on } c \text { during } \tau) \\
& =\prod_{\substack{w \in \mathcal{N}(u, c) \\
w \neq v}}\{1-\operatorname{Pr}(w \text { transmits on } c \text { during } \tau)\} \\
& =\prod_{\substack{w \in \mathcal{N}(u, c) \\
w \neq v}}\left\{1-\frac{1}{|\mathcal{A}(w)|} \times \min \left(\frac{1}{2}, \frac{|\mathcal{A}(w)|}{2^{k}}\right)\right\} \\
& =\prod_{\substack{w \in \mathcal{N}(u, c) \\
w \neq v}}\left\{1-\min \left(\frac{1}{2|\mathcal{A}(w)|}, \frac{1}{2^{k}}\right)\right\} \\
& \{\min (x, y) \leq y\} \\
& \geq \prod_{\substack{w \in \mathcal{N}(u, c) \\
w \neq v}}\left(1-\frac{1}{2^{k}}\right) \\
& =\left(1-\frac{1}{2^{k}}\right)^{|\mathcal{N}(u, c)|-1} \\
& \left\{|\mathcal{N}(u, c)|-1=\Delta(u, c)-1 \text { and, using (2), } \Delta(u, c)-1 \leq 2^{k}\right\} \\
& \geq\left(1-\frac{1}{2^{k}}\right)^{2^{k}}
\end{aligned}
$$




$$
\begin{aligned}
& \left\{\forall x \geq 2,\left(1-\frac{1}{x}\right)^{x} \text { is a monotonically increasing function of } x \text { and thus } \geq \frac{1}{4}\right\} \\
\geq & \frac{1}{4}
\end{aligned}
$$

\section{A.4 Computing the probability that $s$ covers $(v, u)$}

Let $F(\tau, c)$ denote the event that $\tau$ covers $(v, u)$ on $c$. Note that $F(\tau, c)=A(\tau, c) \wedge B(\tau, c) \wedge C(\tau, c)$. Since $A(\tau, c), B(\tau, c)$ and $C(\tau, c)$ are mutually independent events, $\operatorname{Pr}\{F(\tau, c)\}=\operatorname{Pr}\{A(\tau, c)\} \times \operatorname{Pr}\{B(\tau, c)\} \times$ $\operatorname{Pr}\{C(\tau, c)\}$. We have:

$$
\begin{aligned}
\operatorname{Pr}\{F(\tau, c)\} & \geq \frac{1}{2 \max (S, \Delta)} \times \frac{1}{2|\mathcal{A}(u)|} \times \frac{1}{4} \\
& =\frac{1}{16|\mathcal{A}(u)| \max (S, \Delta)}
\end{aligned}
$$

Now, we have:

$$
\begin{aligned}
& \operatorname{Pr}\{s \text { covers }(v, u)\} \\
= & \sum_{c} \operatorname{Pr}\{s \text { covers }(v, u) \text { on } c\} \\
= & \sum_{c \in \mathcal{A}(v) \cap \mathcal{A}(u)} \operatorname{Pr}\{s \text { covers }(v, u) \text { on } c\}+\sum_{c \notin \mathcal{A}(v) \cap \mathcal{A}(u)} \operatorname{Pr}\{s \text { covers }(v, u) \text { on } c\} \\
& \{c \notin \mathcal{A}(v) \cap \mathcal{A}(u) \text { implies that } \operatorname{Pr}\{s \text { covers }(v, u) \text { on } c\}=0\} \\
= & \sum_{c \in \mathcal{A}(v) \cap \mathcal{A}(u)} \operatorname{Pr}\{s \text { covers }(v, u) \text { on } c\} \\
\geq & \sum_{c \in \mathcal{A}(v) \cap \mathcal{A}(u)} \operatorname{Pr}\{F(\tau, c)\} \\
\geq & \sum_{c \in \mathcal{A}(v) \cap \mathcal{A}(u)} \frac{1}{16|\mathcal{A}(u)| \max (S, \Delta)} \\
& =\frac{|\mathcal{A}(v) \cap \mathcal{A}(u)|}{|\mathcal{A}(u)|} \times \frac{1}{16 \max (S, \Delta)} \geq \frac{\rho}{16 \max (S, \Delta)}
\end{aligned}
$$

A.5 Computing the probability of successful discovery of a link in $\frac{16 \max (S, \Delta)}{\rho} \ln \left(\frac{N^{2}}{\epsilon}\right)$ stages Let $M=\frac{16 \max (S, \Delta)}{\rho} \ln \left(\frac{N^{2}}{\epsilon}\right)$. We have:

$$
\operatorname{Pr}((v, u) \text { is not covered within } M \text { stages })
$$




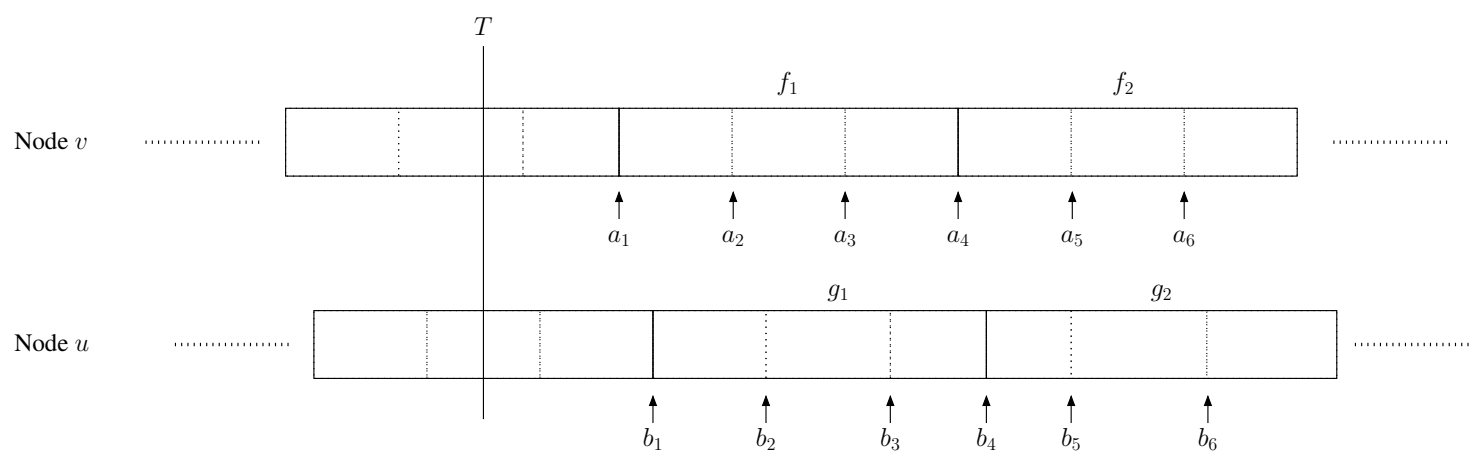

Figure 4: Execution of nodes $v$ and $u$ starting at time $T$. In the figure, $a_{i}$, for $i=1,2, \ldots, 6$, denotes the start time of the six time-slots of the first two full frames of $v$. Likewise, $b_{i}$, for $i=1,2, \ldots, 6$, is the start time of the six time-slots of the first two full frames of $u$.

$$
\begin{aligned}
= & \prod_{1 \leq i \leq M} \operatorname{Pr}\left((v, u) \text { is not covered in the } i^{\text {th }} \text { stage }\right) \\
\leq & \prod_{1 \leq i \leq M}\left(1-\frac{\rho}{16 \max (S, \Delta)}\right) \\
= & \left(1-\frac{\rho}{16 \max (S, \Delta)}\right)^{\frac{16 \max (S, \Delta)}{\rho} \ln \left(\frac{N^{2}}{\epsilon}\right)} \\
& \left\{\forall x \geq 2,\left(1-\frac{1}{x}\right)^{x} \text { is upper bounded by } \frac{1}{e}\right\} \\
\leq & \left(\frac{1}{e}\right)^{\ln \left(\frac{N^{2}}{\epsilon}\right)}=\frac{\epsilon}{N^{2}}
\end{aligned}
$$

\section{B Omitted Proof of Lemma 8 in Section 4.1}

Note that the frames $f_{1}$ and $f_{2}$ together contain six slots. Let $a_{i}$ for $i=1,2, \ldots, 6$ denote the start times of the six slots numbered in the increasing order of their start times (see Fig. 4). Likewise, let $b_{i}$ for $i=1,2, \ldots, 6$ denote the start times of the six slots of the frames $g_{1}$ and $g_{2}$.

Claim 1: We first show that $b_{1} \leq a_{5}$. Assume, by the way of contradiction, that $a_{5}<b_{1}$. Note that, by definition of $g_{1}$, there is only a partial frame of $u$ between $T$ and $b_{1}$. This, in turn, implies that a partial frame of $u$ contains at least four slots of $v$. This can happen only if the following condition holds:

$$
\frac{L}{1-\delta}>\frac{4 L}{3(1+\delta)} \quad \Longrightarrow \quad \delta>\frac{1}{7}
$$

This contradicts Assumption 1. 


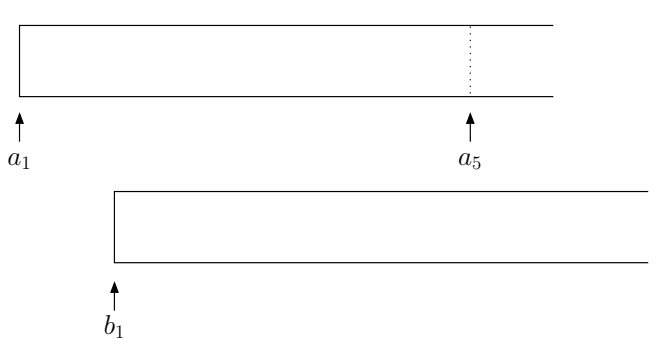

(a)

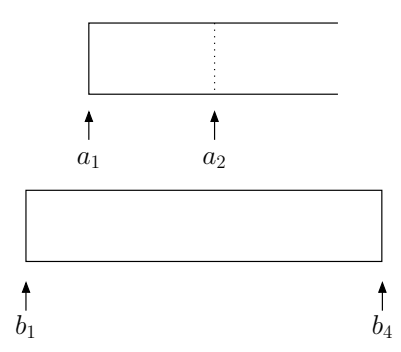

(b)

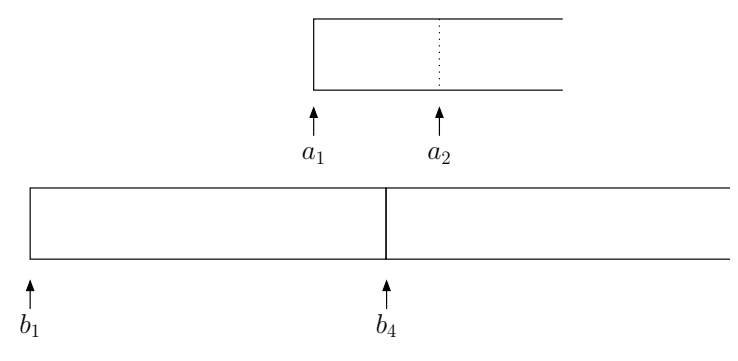

(c)

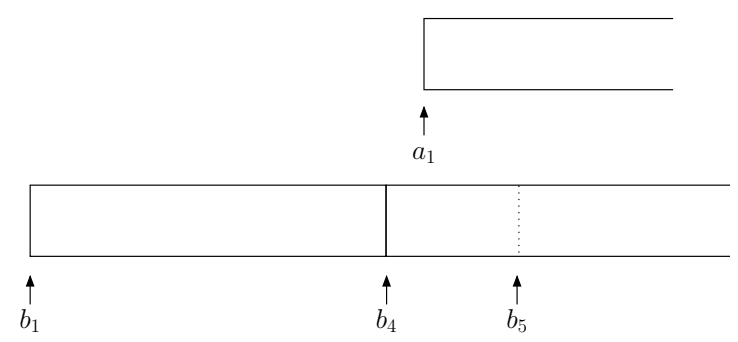

(d)

Figure 5: Various cases in the proof of Lemma 8: (a) $a_{1}<b_{1}$, (b) $b_{1} \leq a_{1}$ and $a_{2} \leq b_{4}$, (c) $b_{1} \leq a_{1}<$ $a_{2}<b_{4}$, and (d) $b_{4}<a_{1}$.

Claim 2: Likewise, we can show that $a_{1} \leq b_{5}$.

Claim 3: If $a_{i-1} \leq b_{1} \leq a_{i}$ for some $i$ with $1<i \leq 5$, then $b_{1} \leq a_{i}<a_{i+1} \leq b_{4}$. It suffices to show that $a_{i+1} \leq b_{4}$. Assume, by the way of contradiction, that $b_{4}<a_{i+1}$. This implies that $a_{i-1} \leq b_{1}<b_{4}<a_{i+1}$. In other words, two adjacent slots of $v$ strictly contain an entire frame of $u$. This can happen only if the following condition holds:

$$
\frac{2 L}{3(1-\delta)}>\frac{L}{1+\delta} \quad \Longrightarrow \quad \delta>\frac{1}{5}
$$

This contradicts Assumption 1.

We now prove the lemma statement. We consider two cases depending on whether $f_{1}$ or $g_{1}$ has earlier start time.

- Case $1\left(a_{1}<b_{1}\right)$ : In this case, we have $a_{1}<b_{1} \leq a_{5}$ (see Fig. 5(a)). The rightmost inequality follows from Claim 1. The time interval from $a_{1}$ to $a_{5}$ consists of four contiguous slots of $v:\left[a_{1}, a_{2}\right]$, $\left[a_{2}, a_{3}\right],\left[a_{3}, a_{4}\right]$ and $\left[a_{4}, a_{5}\right]$. Clearly, $b_{1}$ lies in at least one of them. (If $b_{1}$ lies on the boundary of two 
slots, we select the earlier one.) In any case, using Claim 3, we can show that at least one of the slots $\left[a_{2}, a_{3}\right],\left[a_{3}, a_{4}\right],\left[a_{4}, a_{5}\right]$ or $\left[a_{5}, a_{6}\right]$ is contained in the frame $g_{2}$. This implies that either $\left\langle f_{1}, g_{1}\right\rangle$ or $\left\langle f_{2}, g_{1}\right\rangle$ is aligned.

- Case $2\left(b_{1} \leq a_{1}\right)$ : If $a_{2} \leq b_{4}$, then $b_{1} \leq a_{1}<a_{2} \leq b_{4}$ (see Fig. 5(b)). In other words, the first slot of the frame $f_{1}$ is contained within the frame $g_{1}$, which implies that $\left\langle f_{1}, g_{1}\right\rangle$ is aligned. Therefore assume that $b_{4}<a_{2}$. We have two subcases depending on where $a_{1}$ lies relative to $b_{4}$. Let $b_{7}$ denote the end time of the frame $g_{2}$.

- Case $2.1\left(a_{1} \leq b_{4}\right)$ : In this case, we show that the slot $\left[a_{2}, a_{3}\right]$ is contained within the frame $\left[b_{4}, b_{7}\right]$ (see Fig. 5(c)). To that end, we first prove that $a_{3}<b_{7}$. If not, then $a_{1} \leq b_{4}<b_{7} \leq a_{3}$. In other words, two adjacent slots of $v$ strictly contain an entire frame of $u$. This can happen only if the following condition holds:

$$
\frac{2 L}{3(1-\delta)}>\frac{L}{1+\delta} \quad \Longrightarrow \quad \delta>\frac{1}{5}
$$

This contradicts Assumption 1. Therefore, we have $b_{4}<a_{2}<a_{3}<b_{7}$, which implies that the pair $\left\langle f_{1}, g_{2}\right\rangle$ is aligned.

- Case $2.2\left(b_{4}<a_{1}\right):$ In this case, we show that the slot $\left[a_{1}, a_{2}\right]$ is contained within the frame $\left[b_{4}, b_{7}\right]$ (see Fig. 5(d)). To that end, we show that $a_{2} \leq b_{7}$. If not, then $a_{1} \leq b_{5}<b_{7}<a_{2}$. (The inequality $a_{1} \leq b_{5}$ follows from Claim 2.) In other words, one slot of $v$ strictly contains at least two adjacent slots of $u$. This can happen only if the following condition holds:

$$
\frac{L}{3(1-\delta)}>\frac{2 L}{3(1+\delta)} \quad \Longrightarrow \quad \delta>\frac{1}{3}
$$

This contradicts Assumption 1. Therefore, we have $b_{4}<a_{1}<a_{2} \leq b_{7}$, which implies that the pair $\left\langle f_{1}, g_{2}\right\rangle$ is aligned.

In all cases, we show that one of the four pairs $\left\langle f_{1}, g_{1}\right\rangle,\left\langle f_{1}, g_{2}\right\rangle,\left\langle f_{2}, g_{1}\right\rangle$ or $\left\langle f_{2}, g_{2}\right\rangle$ is aligned. 


\section{Omitted Proof from Section 4.2}

Computing the sum of the number of time-slots/frames in the first $k$ epochs: Let $S$ denote the sum $\sum_{1 \leq i \leq k}(i+1) \cdot 2^{i}$. We have:

$$
\begin{aligned}
S= & 2 \cdot 2^{1}+3 \cdot 2^{2}+4 \cdot 2^{3}+\cdots+(k+1) \cdot 2^{k} \\
2 S= & 2 \cdot 2^{2}+3 \cdot 2^{3}+\cdots+2^{k}+(k+1) \cdot 2^{k+1}
\end{aligned}
$$

Subtracting the first equation from the second and rearranging terms, we obtain:

$$
\begin{aligned}
S & =(k+1) \cdot 2^{k+1}-\left(2^{k}+2^{k-1}+\cdots+2^{2}\right)-2 \cdot 2^{1} \\
& =(k+1) \cdot 2^{k+1}-\left(\sum_{1 \leq i \leq k} 2^{i}\right)-2 \\
& =(k+1) \cdot 2^{k+1}-2 \cdot\left(2^{k}-1\right)-2 \\
& =k \cdot 2^{k+1}
\end{aligned}
$$

\section{Omitted Proofs from Section 6.3.1}

\section{D.1 Computing the conditional probability of occurrence of $F(\tau, c, w)$ given $A B C(\tau, c)$}

First, assume that $w \in G_{2}$. We have:

$$
\begin{aligned}
\operatorname{Pr}\{F(\tau, c, w) \mid A B C(\tau, c)\}= & \operatorname{Pr}\{w \text { does not transmit on } \mathcal{A} \backslash\{c, j\} \mid w \text { does not transmit on } c\} \\
& \left\{\operatorname{Pr}\{X \mid Y\}=\frac{\operatorname{Pr}\{X \wedge Y\}}{\operatorname{Pr}\{Y\}}\right\} \\
= & \frac{\operatorname{Pr}\{(w \text { does not transmit on } \mathcal{A} \backslash\{c, j\}) \wedge(w \text { does not transmit on } c)\}}{\operatorname{Pr}\{w \text { does not transmit on } c\}} \\
= & \frac{\operatorname{Pr}\{(w \text { listens }) \text { or }(w \text { transmits on channel } j)\}}{\operatorname{Pr}\{(w \text { listens }) \text { or }(w \text { transmits on a channel other than } c)\}} \\
= & \frac{1-p+p \cdot \frac{1}{S}}{1-p+p \cdot \frac{S-1}{S}}=\frac{1-p \frac{S-1}{S}}{1-\frac{p}{S}} \\
& \left\{\text { multiplying numerator and denominator by } \frac{S}{p}\right\} \\
= & \frac{\frac{S}{p}-S+1}{\frac{S}{p}-1}=1-\frac{S-2}{\frac{S}{p}-1}
\end{aligned}
$$


Next, assume that $w \in G_{3}$. We have:

$$
\begin{aligned}
\operatorname{Pr}\{F(\tau, c, w) \mid A B C(\tau, c)\} & =\operatorname{Pr}\{w \text { does not transmit on } \mathcal{A} \backslash\{c, j\}\} \\
& =\operatorname{Pr}\{(w \text { listens }) \text { or }(w \text { transmits on channel } c \text { or } j)\} \\
& =1-p+p \cdot \frac{2}{S}=1-p \cdot \frac{S-2}{S}=1-\frac{S-2}{\frac{S}{p}}
\end{aligned}
$$

\section{D.2 Computing the conditional probability of occurrence of $D(\tau, c)$ given $A B C(\tau, c)$}

We analyze the cases corresponding to when $p=\frac{1}{2}$ and when $p=\frac{S}{\Delta_{e s t}}$ separately.

- Case $1\left(p=\frac{1}{2}\right)$ : Using (18) and (22), we have:

$$
\begin{aligned}
\operatorname{Pr}\{D(\tau, c) \mid A B C(\tau, c)\} \geq & \frac{1}{2} \cdot\left(\frac{S-1}{S}\right) \cdot\left\{1-\left(1-\frac{S-2}{2 S}\right)^{N-2}\right\} \\
& \{N \geq 3\} \\
\geq & \frac{1}{2} \cdot\left(\frac{S-1}{S}\right) \cdot\left(\frac{S-2}{2 S}\right)=\frac{1}{4} \cdot\left(1-\frac{1}{S}\right) \cdot\left(1-\frac{2}{S}\right) \\
& \{S \geq 3\} \\
\geq & \frac{1}{4} \cdot \frac{2}{3} \cdot \frac{1}{3}=\frac{1}{18}
\end{aligned}
$$

- Case $2\left(p=\frac{S}{\Delta_{e s t}}\right)$ : Using (22), we have:

$$
\begin{aligned}
\operatorname{Pr}\{E(\tau, c) \mid A B C(\tau, c)\}= & 1-\left(1-\frac{S-2}{\Delta_{\text {est }}}\right)^{N-2} \\
& \left\{\text { substitute } x=\frac{\Delta_{\text {est }}}{S-2}\right. \\
\left.p=\frac{S}{\Delta_{\text {est }}} \Longrightarrow \frac{S}{\Delta_{\text {est }}} \leq \frac{1}{2} \Longrightarrow \frac{\Delta_{\text {est }}}{S} \geq 2 \Longrightarrow x \geq 2\right\} & 1-\left(1-\frac{1}{x}\right)^{N-2} \\
\geq & \left\{\text { using }(20), N-2 \geq \frac{\Delta_{\text {est }}}{2} \Longrightarrow N-2 \geq \frac{x(S-2)}{2}\right\} \\
\geq & 1-\left\{\left(1-\frac{1}{x}\right)^{x}\right\}^{\frac{S-2}{2}} \\
& \left\{\left(1-\frac{1}{x}\right)^{x} \leq \frac{1}{\mathrm{e}} \text { when } x \geq 2\right\}
\end{aligned}
$$




$$
\geq 1-\left(\frac{1}{\mathrm{e}}\right)^{\frac{S-2}{2}}
$$

Using (18) and (23), we have:

$$
\begin{aligned}
\operatorname{Pr}\{D(\tau, c) \mid A B C(\tau, c)\} & \geq \frac{1}{2} \cdot\left(\frac{S-1}{S}\right) \cdot\left\{1-\left(\frac{1}{\mathrm{e}}\right)^{\frac{S-2}{2}}\right\} \\
& \geq \frac{1}{2} \cdot\left(1-\frac{1}{S}\right) \cdot\left(1-\frac{1}{\sqrt{\mathrm{e}}}\right) \\
& \{S \geq 3\} \\
\geq & \frac{1}{2} \cdot \frac{2}{3} \cdot \frac{1}{3}=\frac{1}{9}
\end{aligned}
$$

D.3 Computing the conditional probability of occurrence of $D(\tau, c)$ given $A B C(\tau, c)$ when $N$ and $S$ are sufficiently large

- Case $1\left(p=\frac{1}{2}\right)$ : Using (18) and (22) we have:

$$
\begin{aligned}
\operatorname{Pr}\{D(\tau, c) \mid A B C(\tau, c)\} \geq & \frac{1}{2} \cdot\left(\frac{S-1}{S}\right) \cdot\left\{1-\left(1-\frac{S-2}{2 S}\right)^{N-2}\right\} \\
& \left\{\text { for sufficiently large } S, \frac{S-1}{S} \approx 1 \text { and } \frac{S-2}{2 S} \approx \frac{1}{2}\right\} \\
& \frac{1}{2} \cdot 1 \cdot\left\{1-\left(\frac{1}{2}\right)^{N-2}\right\} \\
& \left\{\text { for sufficiently large } N,\left(\frac{1}{2}\right)^{N-2} \approx 0\right\} \\
& \frac{1}{2} \cdot(1-0)=\frac{1}{2}
\end{aligned}
$$

- Case $2\left(p=\frac{S}{\Delta_{e s t}}\right)$ : Using (18) and (23), we have:

$$
\begin{aligned}
\operatorname{Pr}\{D(\tau, c) \mid A B C(\tau, c)\} \geq & \frac{1}{2} \cdot\left(\frac{S-1}{S}\right) \cdot\left\{1-\left(\frac{1}{\mathrm{e}}\right)^{\frac{S-2}{2}}\right\} \\
& \left\{\text { for sufficiently large } S, \frac{S-1}{S} \approx 1 \text { and }\left(\frac{1}{\mathrm{e}}\right)^{\frac{S-2}{2}} \approx 0\right\} \\
\approx & \frac{1}{2} \cdot 1 \cdot(1-0)=\frac{1}{2}
\end{aligned}
$$




\section{E Omitted Proofs from Section 6.3.2}

\section{E.1 Computing the probability of occurrence of $\hat{C}^{\prime}(f, g, c)$}

We focus on the case when exactly three slots of $w$ overlap with $\operatorname{slot}(f, g)$. But the result also holds if fewer than three slots of $w$ overlap with $\operatorname{slot}(f, g)$. There are two sub-cases: the three (overlapping) slots belong to the same frame or two different frames.

Sub-case 1 (three slots belong to the same frame): In this case, we have:

$$
\begin{aligned}
\operatorname{Pr}\{w \text { does not transmit on } c \text { during } \operatorname{slot}(f, g)\}\} & =1-p+p \cdot\left(\frac{S-1}{S}\right)^{3} \\
& =1-p\left(1-\frac{(S-1)^{3}}{S^{3}}\right) \\
& =1-p\left(\frac{3 S^{2}-3 S+1}{S^{3}}\right) \\
& \geq 1-p\left(\frac{3 S^{2}}{S^{3}}\right) \\
& \geq 1-\left(\frac{S}{3 \Delta_{e s t}}\right) \cdot\left(\frac{3}{S}\right) \\
& =1-\frac{1}{\Delta_{\text {est }}}
\end{aligned}
$$

Sub-case 2 (the three slots come from two different frames): In this case, we have:

$$
\begin{aligned}
\operatorname{Pr}\{w \text { does not transmit on } c \text { during } \operatorname{slot}(f, g)\} & =\left\{1-p+p \cdot\left(\frac{S-1}{S}\right)\right\} \cdot\left\{1-p+p \cdot\left(\frac{S-1}{S}\right)^{2}\right\} \\
& =\left(1-p \cdot \frac{1}{S}\right) \cdot\left(1-p \cdot \frac{2 S-1}{S^{2}}\right) \\
& =1-p \cdot \frac{1}{S}-p \cdot \frac{2 S-1}{S^{2}}+p^{2} \cdot \frac{2 S-1}{S^{3}} \\
& \geq 1-p \cdot \frac{3 S-1}{S^{2}} \\
& \geq 1-p \cdot \frac{3 S}{S^{2}} \\
& =1-p \cdot \frac{3}{S} \\
& \geq 1-\frac{S}{3 \Delta_{e s t}} \cdot \frac{3}{S}=1-\frac{1}{\Delta_{e s t}}
\end{aligned}
$$

It can now be shown, in a similar manner as in the proof of Lemma 6 , that $\operatorname{Pr}\left\{\hat{C}^{\prime}(f, g, c)\right\} \geq \frac{1}{4}$. 
E.2 Computing the conditional probability of occurrence of $\hat{D}(f, g, c)$ given $A \hat{B} C(f, g, c)$ when $p=\frac{S}{3 \Delta_{e s t}}$

Using an expression similar to (22), we can show the following:

$$
\operatorname{Pr}\{\hat{E}(f, g, c) \mid A \hat{B} C(f, g, c)\}=1-\left(1-\frac{S-2}{3 \Delta_{\text {est }}}\right)^{N-2} \geq 1-\left(\frac{1}{\mathrm{e}}\right)^{\frac{S-2}{6}}
$$

For arbitrary $N$ and $S$ with $N, S \geq 3$, we have:

$$
\begin{aligned}
\operatorname{Pr}\{\hat{D}(f, g, c) \mid A \hat{B} C(f, g, c)\} & \geq \frac{1}{2} \cdot\left(\frac{S-1}{S}\right) \cdot\left\{1-\left(\frac{1}{\mathrm{e}}\right)^{\frac{S-2}{6}}\right\} \\
& \geq \frac{1}{2} \cdot\left(1-\frac{1}{S}\right) \cdot\left(1-\frac{1}{\sqrt[6]{\mathrm{e}}}\right) \\
& \{S \geq 3\} \\
& =\frac{1}{2} \cdot \frac{2}{3} \cdot \frac{1}{7}=\frac{1}{21}
\end{aligned}
$$

For sufficiently large $N$ and $S$, we have:

$$
\begin{aligned}
\operatorname{Pr}\{\hat{D}(f, g, c) \mid A \hat{B} C(f, g, c)\} & \geq \frac{1}{2} \cdot\left(\frac{S-1}{S}\right) \cdot\left\{1-\left(\frac{1}{\mathrm{e}}\right)^{\frac{S-2}{6}}\right\} \\
& \gtrsim \frac{1}{2} \cdot 1 \cdot(1-0)=\frac{1}{2}
\end{aligned}
$$


K. Alex Mills is a Ph.D student at The University of Texas at Dallas (UT Dallas). He received both his Bachelor's of Science in Software Engineering (2011) and Master's of Science in Computer Science (2013) from UT Dallas. He is currently working on his dissertation on fault-tolerant replica placement in large-scale data centers. His research interests are social computing, algorithms, combinatorial optimization, and mathematics. 

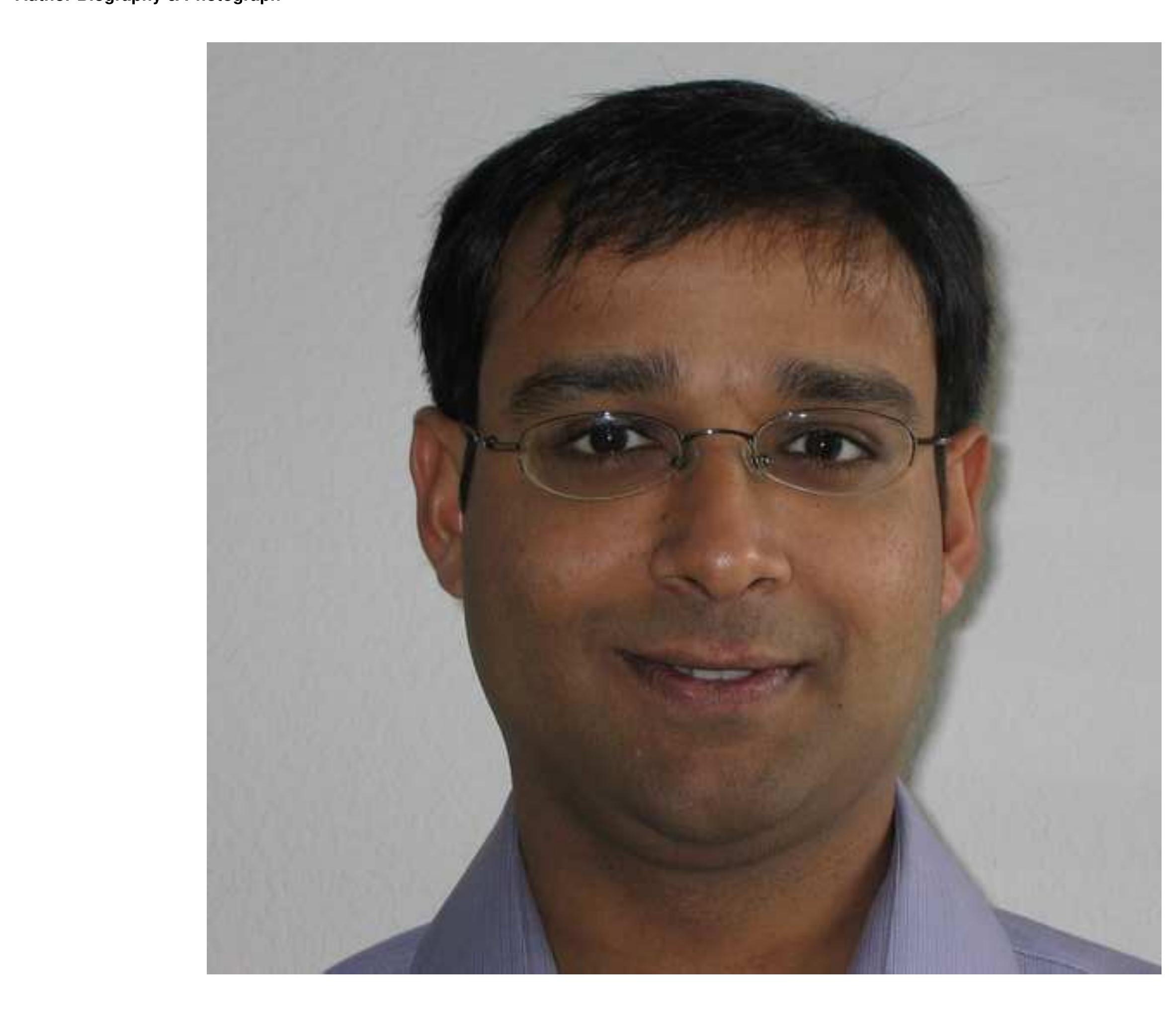
.
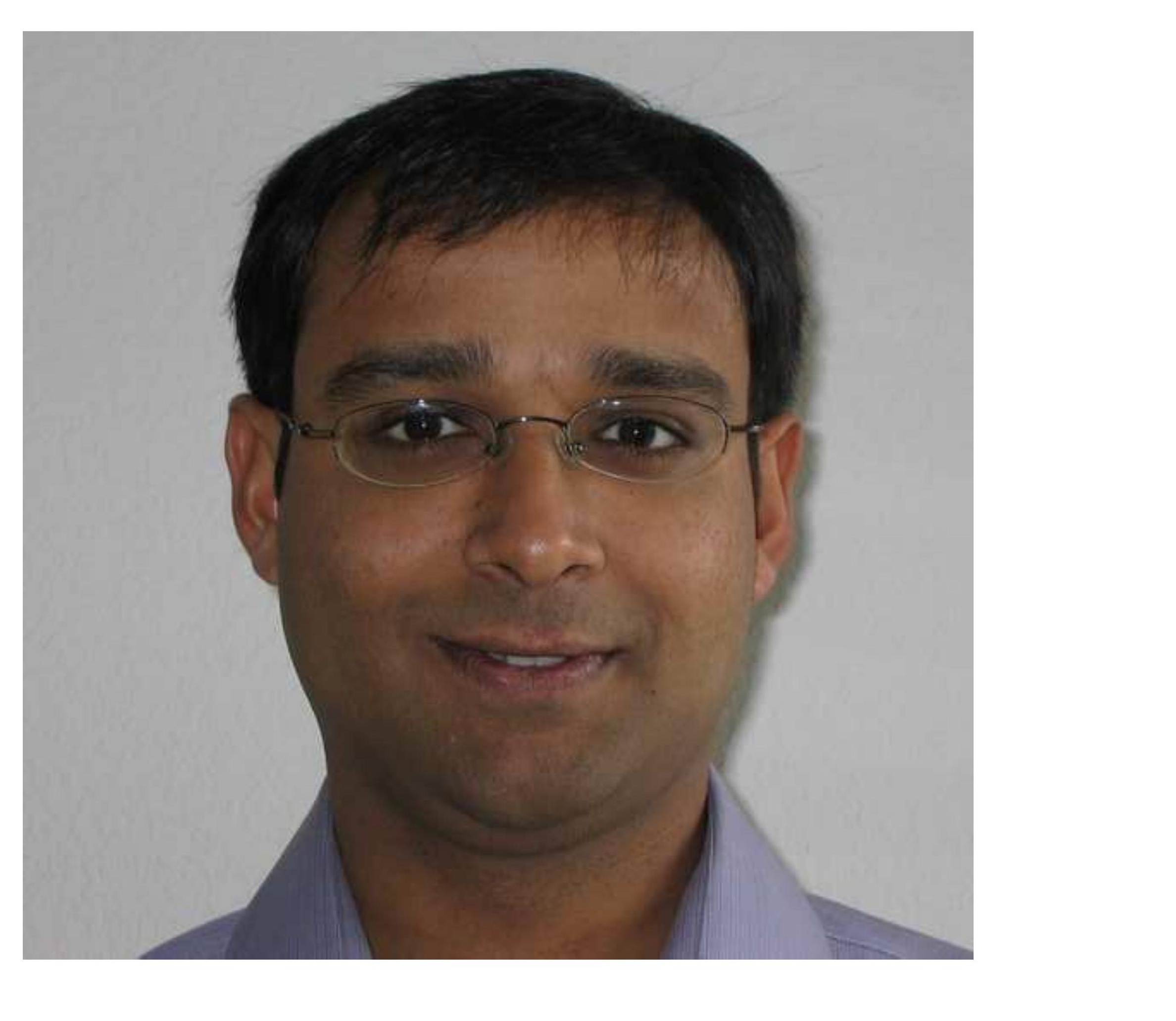


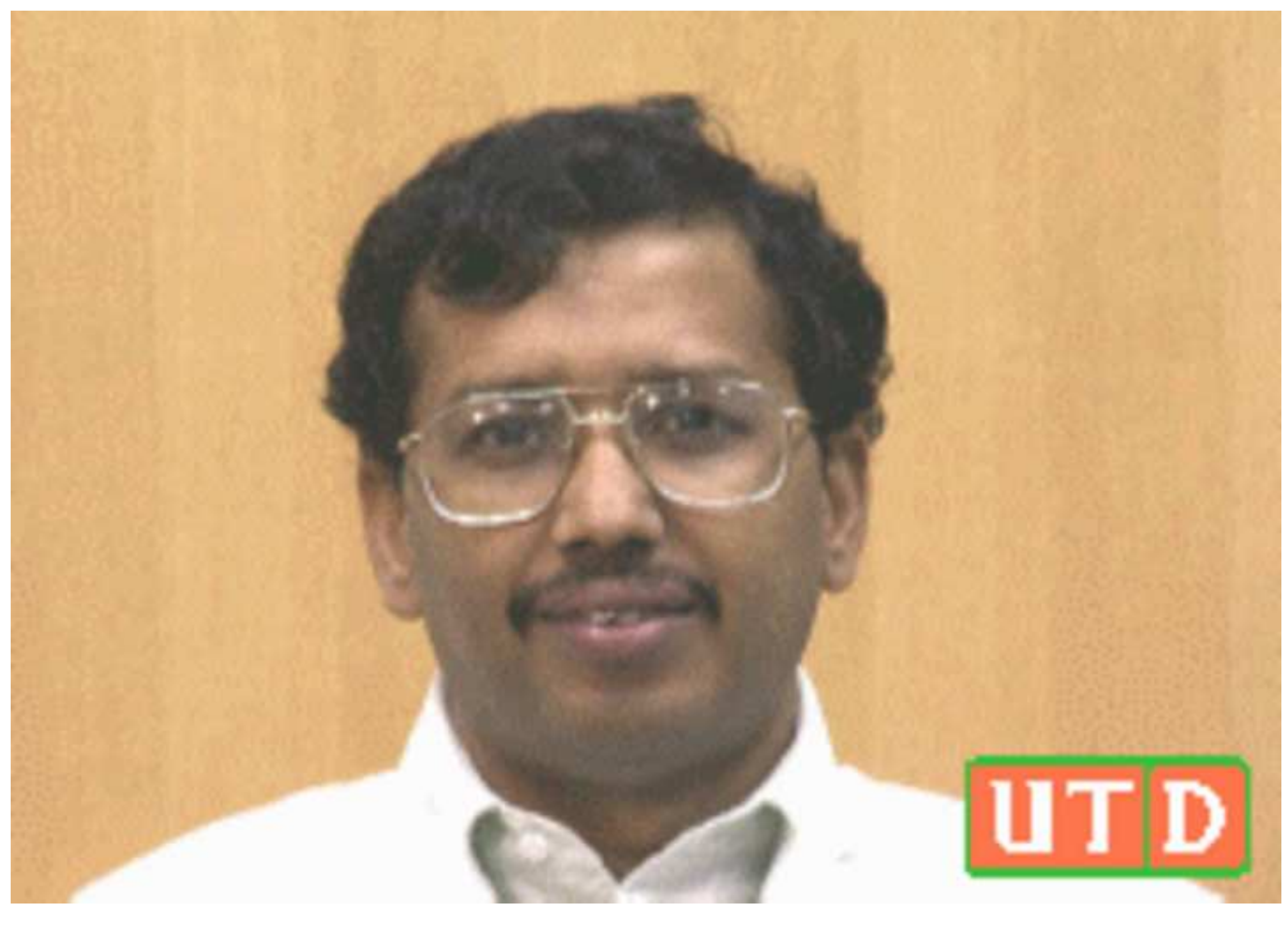

.
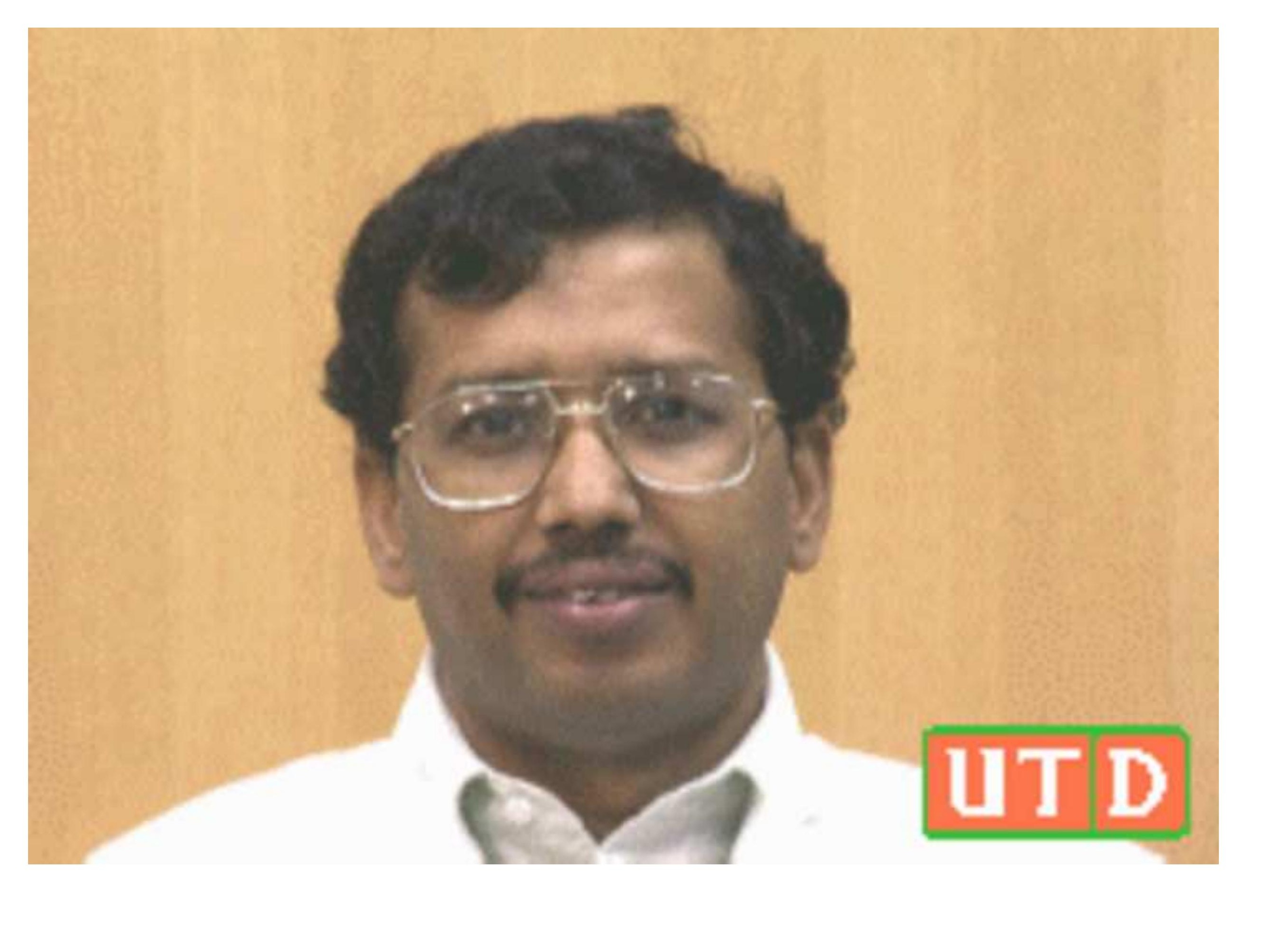


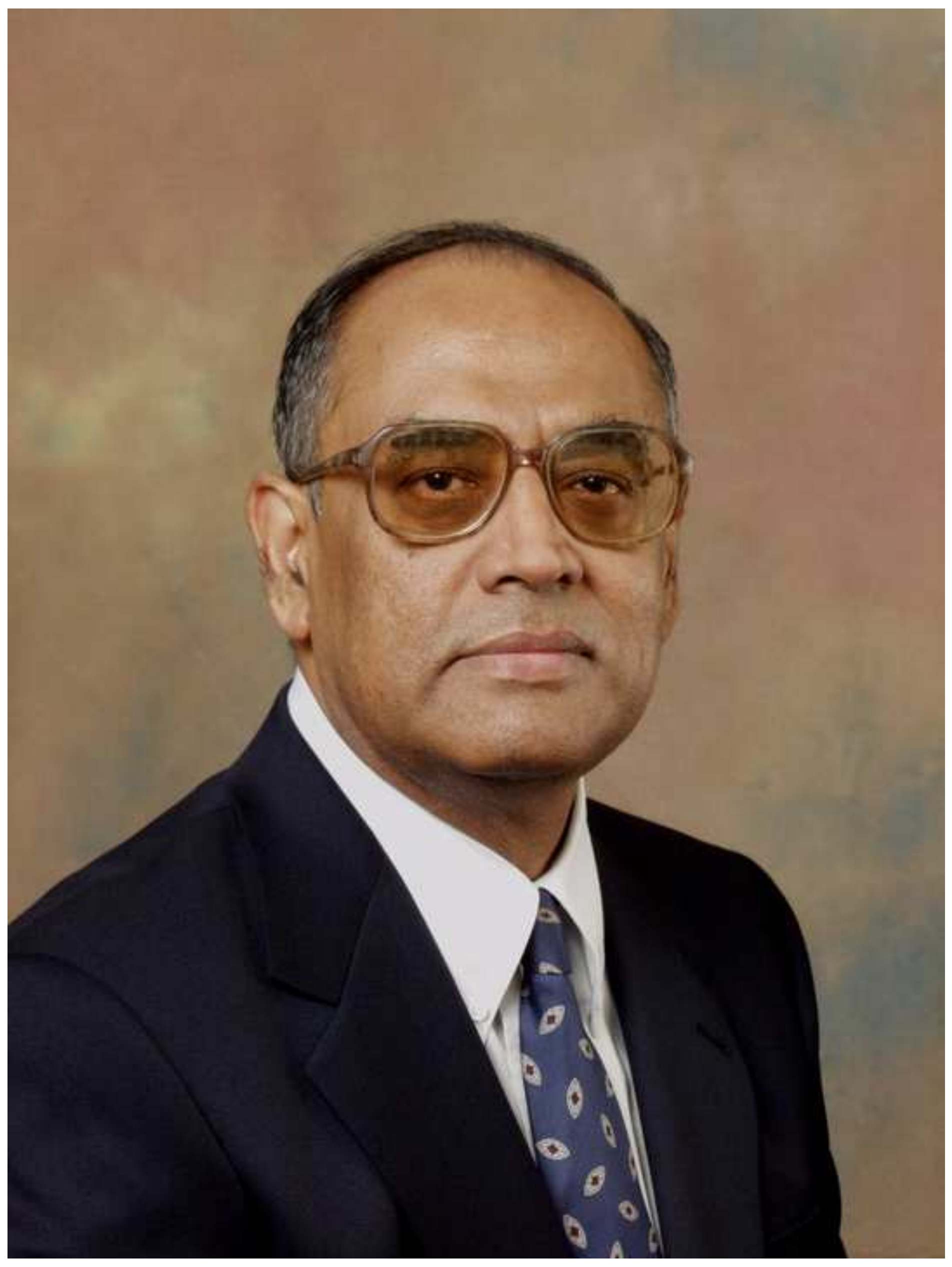

${ }^{*}$ Author Biography \& Photograph

Author Blography \& Photograph

\section{h}


Yanyan Zeng received her B.S. degree in Computer Science from the University of Science and Technology of China, Hefei, in 2004 and her M.S. and Ph.D. degrees in computer science from the University of Texas at Dallas in 2008 and 2011, respectively. She is currently affiliated with Global Technology at JPMorgan Chase \& Co, where she focuses on application and network performance engineering for large scale distributed systems. 
Shreyas Gokhale received his Bachelor of Engineering degree in Computer Engineering from University of Pune, India in 2012 and his M.S. degree in Computer Science from the University of Texas at Dallas in 2014. He is currently pursuing his Ph.D. degree in Computer Science at the Advanced Networking and Dependable Systems Laboratory (ANDES) at the University of Texas at Dallas. His research interests include Distributed Systems, Networking, and Cognitive Radio Networks. 
${ }^{*}$ Author Biography \& Photograph

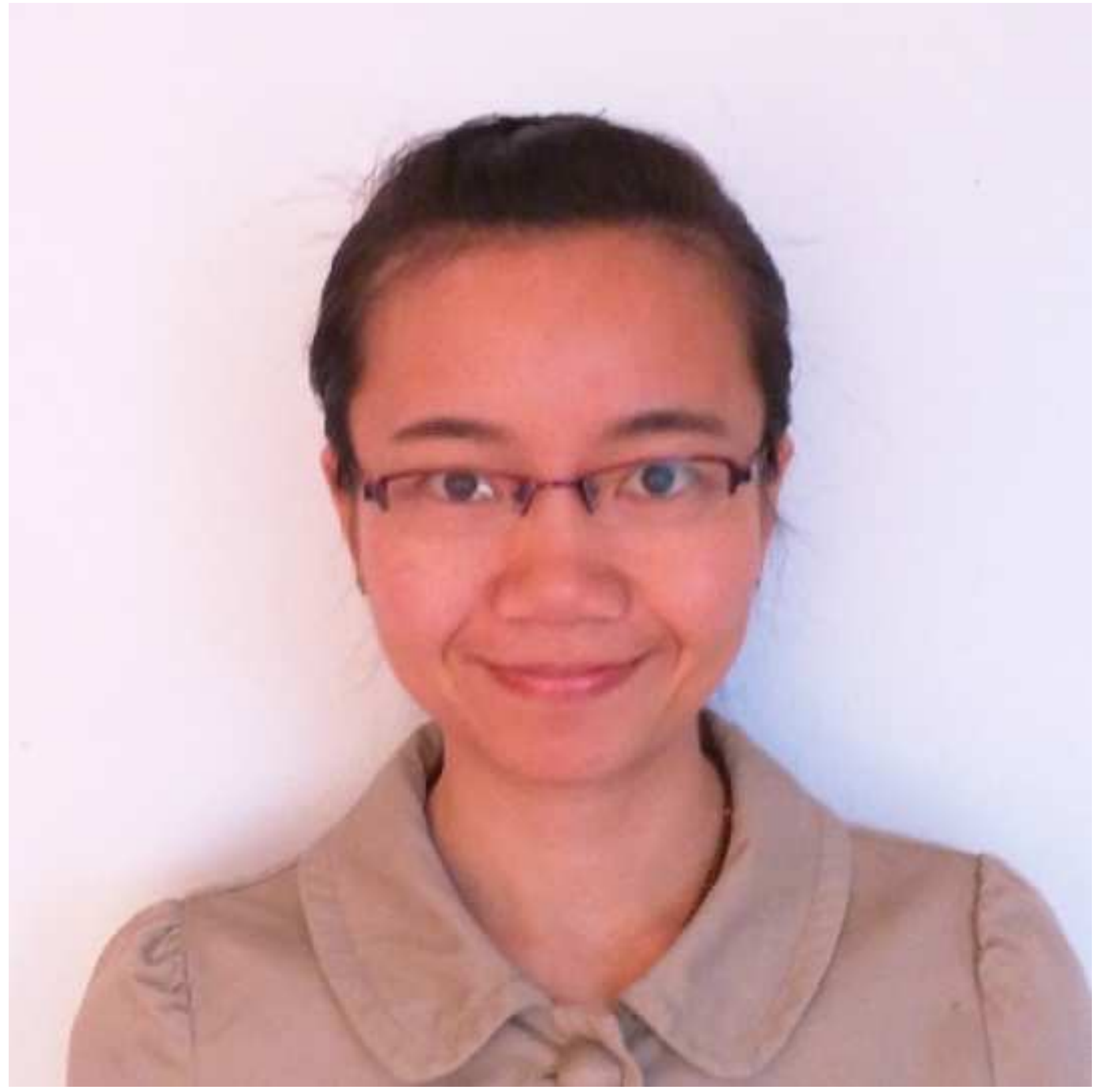




\section{${ }^{*}$ Author Biography \& Photograph}

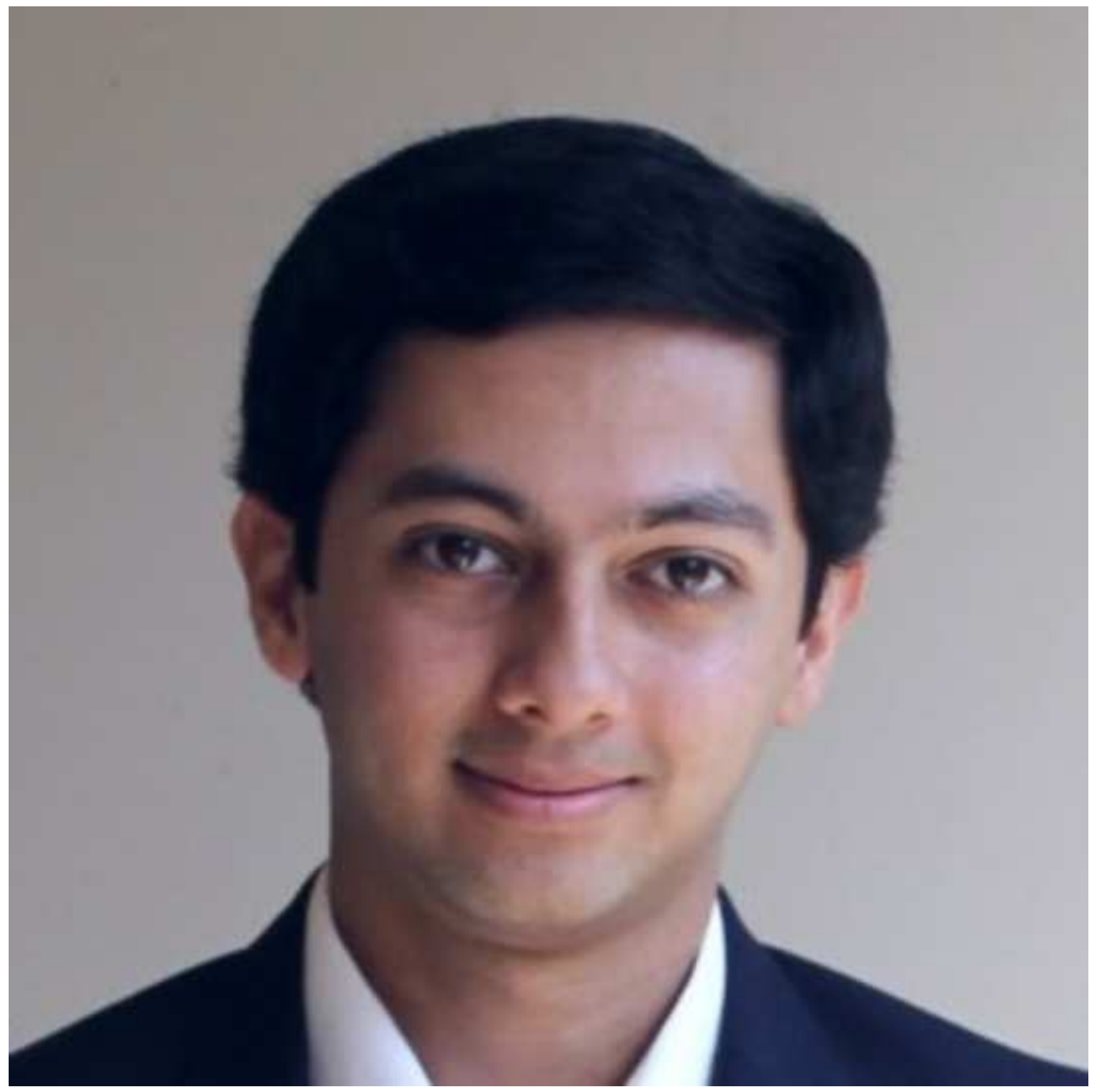


${ }^{\star}$ Author Biography \& Photograph

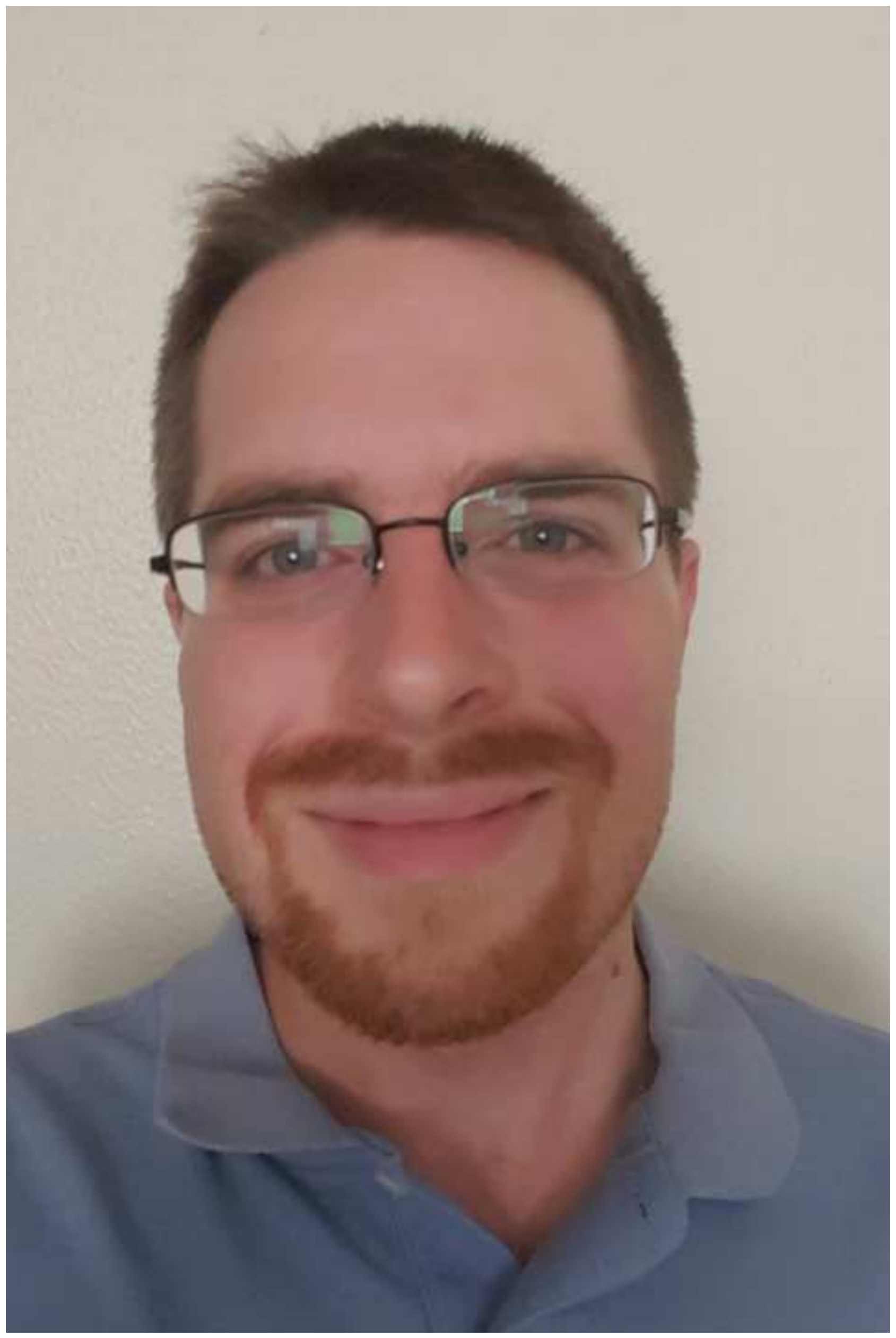


Neeraj Mittal received his B.Tech. degree in Computer Science and Engineering from the Indian Institute of Technology, Delhi in 1995 and M.S. and Ph.D. degrees in Computer Science from the University of Texas atAustin in 1997 and 2002, respectively. He is currently an Associate Professor in the Department of Computer Science at the University of Texas at Dallas and a co-director of the Advanced Networking and Dependable Systems Laboratory (ANDES). His research interests include distributed computing, multi-core computing, distributed algorithms for wireless networking and security. 
S. Venkatesan received his B.Tech. degree in Civil Engineering and M.Tech. degree in Computer Science from the Indian Institute of Technology, Madras in 1981 and 1983, respectively. He completed his Ph.D. degree in Computer Science from the University of Pittsburgh in December 1988. In January 1989, he joined the University of Texas at Dallas where he i s currently a Professor of Computer Science.

His research interests are in Distributed Systems, Sensor Networks, Cognitive Radio Networks, Mobile and

Wireless Networks and Testing and Debugging Distributed Programs.

He has been the Chief Architect of IPmobile (a startup acquired by Cisco Systems in September 2000) and a member of the advisory board of Jahi Networks (a startup acquired by Cisco Systems in December 2004). 
R. Chandrasekaran has a B.Tech. from Indian Institute of Technology, Bombay and a Ph.D. from UC Berkeley. He has been a faculty member at Case Western Reserve, Northwestern and UT Dallas where he is a Professor of Computer Science and Operations Research. His interests include mathematical programming, combinatorial optimization, scheduling and computational geometry. 